\title{
Encoding Two-Dimensional Range Top-k Queries
}

\author{
Seungbum Jo ${ }^{1} \cdot$ Rahul Lingala $^{2} \cdot$ Srinivasa Rao Satti $^{3}$ (D)
}

Received: 24 June 2020 / Accepted: 8 July 2021 / Published online: 27 July 2021

(c) The Author(s) 2021

\begin{abstract}
We consider the problem of encoding two-dimensional arrays, whose elements come from a total order, for answering Top- $k$ queries. The aim is to obtain encodings that use space close to the information-theoretic lower bound, which can be constructed efficiently. For an $m \times n$ array, with $m \leq n$, we first propose an encoding for answering 1-sided Top- $k$ queries, whose query range is restricted to $[1 \ldots m][1 \ldots a]$, for $1 \leq a \leq n$. Next, we propose an encoding for answering for the general (4-sided) Top- $k$ queries that takes $\left(m \lg \left({ }^{(k+1) n}\right)+2 n m(m-1)+o(n)\right)$ bits, which generalizes the joint Cartesian tree of Golin et al. [TCS 2016]. Compared with trivial $O(n m \lg n)$ -bit encoding, our encoding takes less space when $m=o(\lg n)$. In addition to the upper bound results for the encodings, we also give lower bounds on encodings for answering 1 and 4-sided Top- $k$ queries, which show that our upper bound results are almost optimal.
\end{abstract}

Keywords Encoding model $\cdot$ Top- $k$ query $\cdot$ Range minimum query

Preliminary version of these results have appeared in the proceedings of the 27th Annual Symposium on Combinatorial Pattern Matching (CPM 16) [14] and the 29th International Symposium on Algorithms and Computation (ISAAC 2018) [15]

Seungbum Jo was supported by the National Research Foundation of Korea (NRF) grant funded by the Korea government (MSIT) (No. NRF-2020R1G1A1101477).

Srinivasa Rao Satti

srinivasa.r.satti@ntnu.no

Seungbum Jo

sbcho1204@gmail.com

Rahul Lingala

lingalarahul7@gmail.com

1 Chungnam National University, Daejeon, South Korea

2 IIT Bombay, Mumbai, India

3 Norwegian University of Science and Technology, Trondheim, Norway 


\section{Introduction}

Given a one-dimensional (1D) array $A[1 \ldots n]$ of $n$ elements from a total order, the range Top- $k$ query on $A$ (Top- $k(i, j, A), 1 \leq i, j \leq n$ ) returns the positions of $k$ largest values in $A[i \ldots j]$. In this paper, we refer to these queries as 2 -sided Top- $k$ queries; and the special case where the query range is $[1 \ldots i]$, for $1 \leq i \leq n$, as the 1 -sided Top- $k$ queries. We can extend the definition to the twodimensional (2D) case-given an $m \times n$ 2D array $A[1 \ldots m][1 \ldots n]$ of $m n$ elements from a total order and a $k \in\{1, \ldots, m n\}$, the range Top- $k$ query on $A$ (Top- $k(i, j, a, b, A), 1 \leq i, j \leq m, 1 \leq a, b \leq n)$ returns the positions of $k$ largest values in $A[i \ldots j][a \ldots b]$. Without loss of generality, we assume that all elements in $A$ are distinct (by ordering equal elements based on the lexicographic order of their positions). Also, we assume that $m \leq n$. In this paper, we consider the following types of Top- $k$ queries.

- Based on the order in which the answers are reported

- Sorted query: the $k$ positions are reported in sorted order of their corresponding values.

- Unsorted query: the $k$ positions are reported in an arbitrary order.

- Based on the query range

- 1-sided query: the query range is $A[1 \ldots m][1 \ldots b]$, for $1 \leq b \leq n$.

- 4-sided query: the query range is $A[i \ldots j][a \ldots b]$, for $i, j \in\{1, m\}$, and $a, b \in\{1, n\}$.

We consider how to support these range Top- $k$ queries on $A$ in the encoding model. In this model, one needs to construct a data structure (an encoding) so that queries can be answered by only accessing the data structure, without accessing the original input array $A$. The minimum size of such an encoding is also referred to as the effective entropy of the input data [10]. Our aim is to obtain encodings that use space close to the effective entropy, which can be constructed efficiently. In the rest of the paper, we use Top- $k(i, j, a, b)$ to denote Top- $k(i, j, a, b, A)$ if $A$ is clear from the context. Also, unless otherwise mentioned, we assume that all Top- $k$ queries are sorted, and 4-sided Top- $k$ queries. Finally, we assume the standard word-RAM model [17] with word size $\Theta(\lg n)^{1}$.

\subsection{Previous work}

The problem of encoding 1D arrays to support Top- $k$ queries has been widely studied in the recent years. Especially, the case when $k=1$, which is commonly known as the Range maximum query (RMQ) problem, has been studied extensively, and has a wide range of applications [1]. Fischer and Heun [6] proposed an optimal

\footnotetext{
1 We use $\lg n$ to denote $\log _{2} n$.
} 
$2 n+o(n)$-bit data structure which answers RMQ queries on 1D array of size $n$ in constant time. For a 2D array $A$ of size $m \times n$, a trivial way to encode $A$ for answering RMQ queries is to store the rank of all elements in $A$, using $O(n m \lg n)$ bits. Golin et al. [10] show that when $m=2$ and RMQ encodings on each row are given, one can support RMQ queries on $A$ using $n-O(\lg n)$ extra bits by encoding a joint Cartesian tree of the two rows. By extending the above encoding, they obtained $n m(m+3) / 2$-bit encoding for answering RMQ queries on $A$, which takes less space than the trivial $O(n m \lg n)$-bit encoding when $m=o(\lg n)$. Brodal et al. [3] proposed an $O\left(\min \left(n m \lg n, m^{2} n\right)\right)$-bit data structure which supports RMQ queries on $A$ in constant time. Finally, Brodal et al. [2] obtained an optimal $O(\mathrm{~nm} \lg m)$-bit encoding for answering RMQ queries on $A$ (although the queries are not supported efficiently). For the case when $k=2$, Davoodi et al. [5] proposed a 3.272n $+o(n)$-bit data structure to encode a $1 \mathrm{D}$ array of size $n$, which supports Top- 2 queries in constant time. The space was later improved by Gawrychowski and Nicholson [9] to the optimal $2.755 n+o(n)$ bits, although it does not support queries efficiently.

For general $k$, on a 1D array of size $n$, Grossi et al. [11] proposed an $O(n \lg k)$-bit encoding which supports sorted Top- $k$ queries in $O(k)$ time, and showed that at least $n \lg k-O(n)$ bits are necessary for answering 1-sided Top- $k$ queries; Gawrychowski and Nicholson [9] proposed a $(k+1) n H(1 /(k+1))+o(n)$-bit ${ }^{2}$ encoding for Top- $k$ queries (although the queries are not supported efficiently), and showed that at least $(k+1) n H(1 /(k+1))(1-o(1))$ bits are required to encode Top- $k$ queries. They also proposed a $(k+1.5) n H(1.5 /(k+1.5))+o(n \lg k)$-bit data structure for answering Top- $k$ queries in $O\left(k^{6} \lg ^{2} n f(n)\right)$ time, for any strictly increasing function $f$. For a 2D array $A$ of size $m \times n$, one can answer Top- $k$ queries using $O(n m \lg n)$ bits, by storing the rank of all elements in $A$. To support the queries efficiently on this encoding, one can use some of the existing orthogonal range reporting data structures in $3 \mathrm{D}$, in which the $z$-coordinate stores the rank of the elements in $A$ (while $x$ - and $y$-coordinates correspond to the positions of the elements in $A$ ), while reporting the points in sorted order of their ranks. However, all the known 3D orthogonal range reporting data structures use at least linear space (i.e., $O(n m \lg n)$ bits), and take at least $O(k \cdot p o l y \log (n))$ time to answer sorted Top- $k$ queries. See 7 [13] for details. Also Rahul and Tao considered the data structures for answering Top- $k$ queries in $\mathbb{R}^{2}[19,20]$; all their data structures use super-linear space (for 4 -sided queries). To the best of our knowledge, there are no results on encodings for range Top- $k$ queries on $2 \mathrm{D}$ arrays for $k>1$.

\subsection{Our results}

Given an $m \times n$ array $A$, we first show that $O(\min \{n m \lg k, n k \lg m\})$ bits are necessary and sufficient for answering sorted 1-sided Top- $k$ queries. For unsorted 1-sided queries, we show that $O(\min \{n k \lg (m / k), n m \lg (k / m)\})$ bits are necessary and sufficient. This space bound is strictly less than the space used to encode sorted 1-sided

\footnotetext{
${ }^{2} H(x)=x \lg (1 / x)+(1-x) \lg (1 /(1-x))$, i.e., an entropy of the binary string whose density of zero is $x$
} 
Top- $k$ queries. Thus it is interesting to note that in $2 \mathrm{D}$, there is a gap between the space needed to encode the 1-sided Top- $k$ queries for the sorted and unsorted cases. In contrast, in $1 \mathrm{D}$, the space needed to encode the 1-sided Top- $k$ queries for both sorted and unsorted cases are asymptotically the same (for $k=o(n)$ ). We show that such encodings can be simply constructed in $O(m n \lg k+n k)$ time by using a minheap data structure.

Next, in Sect. 3, we consider encodings for 4-sided Top- $k$ queries on an $m \times n$ array $A$. We first observe that one can obtain an $O(m n \lg n)$-bit data structure which answers 4-sided Top- $k$ queries on $A$ in $O(k)$ time, by combining the results of [3] and [4]. We then propose the alternative encoding which uses less space than the trivial $m n\lceil\lg (m n)\rceil$-bit encoding (which stores all the positions of $A$ in sorted order), for small $m$.

To be more precise, we first show that $4 n$ bits are sufficient for answering sorted 4-sided Top- $k$ queries on $2 \times n$ array, when encodings for answering sorted 2 -sided Top- $k$ queries for each row are given. This encoding is obtained by a binary DAG for answering Top-2 queries on $2 \times n$ array which generalizes the $(5 n-O(\lg n))$ -bit encoding of RMQ query on $2 \times n$ array proposed by Golin et al. [10], to general $k$; and shows that we can encode a joint Cartesian tree for general $k$ (which corresponds to the DAG in our paper) using $4 n$ bits. Note that the additional space is independent of $k$. By extending the encoding on $2 \times n$ array, we obtain $\left(m \lg \left(\begin{array}{c}(k+1) n \\ n\end{array}\right)+2 n m(m-1)+o(n)\right)$-bit encoding for answering 4-sided Top- $k$ queries on $m \times n$ arrays. This improves upon the trivial $\lceil m n \log (m n)\rceil$-bit encoding when $m=o(\lg n)$, and also generalizes the $m n(m+3) / 2$-bit encoding [10] for answering RMQ queries. The trivial encoding of the input array takes $O(n m \lg n)$ bits, whereas one can easily show a lower bound of $\Omega(n m \lg (\max (m, k)))$ bits for any encoding of an $m \times n$ array that supports Top- $k$ queries since at least $O(n m \lg m)$ bits are necessary for answering RMQ queries [3], and at least $n \lg k$ bits are necessary for answering Top- $k$ queries for each row [11]. Thus, there is only a small range of parameters where a strict improvement over the trivial encoding is possible. Our result closes this gap partially, achieving a strict improvement when $m=o(\lg n)$.

In Sect. 4, we also obtain a data structure for answering Top- $k$ queries in $O\left(k^{2}+k T(n, k)\right)$ time using $2 S(n, k)+(4 k+7) n+k o(n)$ bits, if there exists an $S(n, k)$-bit encoding to answer sorted 2-sided Top- $k$ queries on a $1 \mathrm{D}$ array of size $n$ in $T(n, k)$ time. Comparing to the $2 S(n, k)+4 n+o(n)$-bit encoding, this data structure uses more space but supports Top- $k$ queries efficiently (the $2 S(n, k)+4 n+o(n)$ -bit encoding takes $O\left(k^{2} n^{2}+n k T(n, k)\right)$ time for answering Top- $k$ queries).

Finally, in Sect. 5 , given a $2 \times n$ array $A$, we consider the lower bound on additional space required to answer unsorted (or sorted) Top- $k$ on $A$ when encodings of Top- $k$ query for each row are given. We show that at least $1.27(n-k / 2)-o(n)$ additional bits are necessary for answering unsorted 1 -sided Top- $k$ queries on $A$, when encodings of unsorted 1 or 2 -sided Top- $k$ query for each row are given. Note that this lower bound also gives the lower bound for answering unsorted 4-sided Top- $k$ queries on $2 \times n$ array under the same condition. We also show that $2 n-O(\lg n)$ additional bits are necessary for answering sorted 4-sided Top- $k$ queries on $A$, when encodings of unsorted (or sorted) 2-sided Top- $k$ query for each row are given. These lower bound results imply that our encodings in 3 are close to optimal (i.e., within 
Table 1 Summary of the results of upper and lower bounds for Top- $k$ encodings on 2D arrays

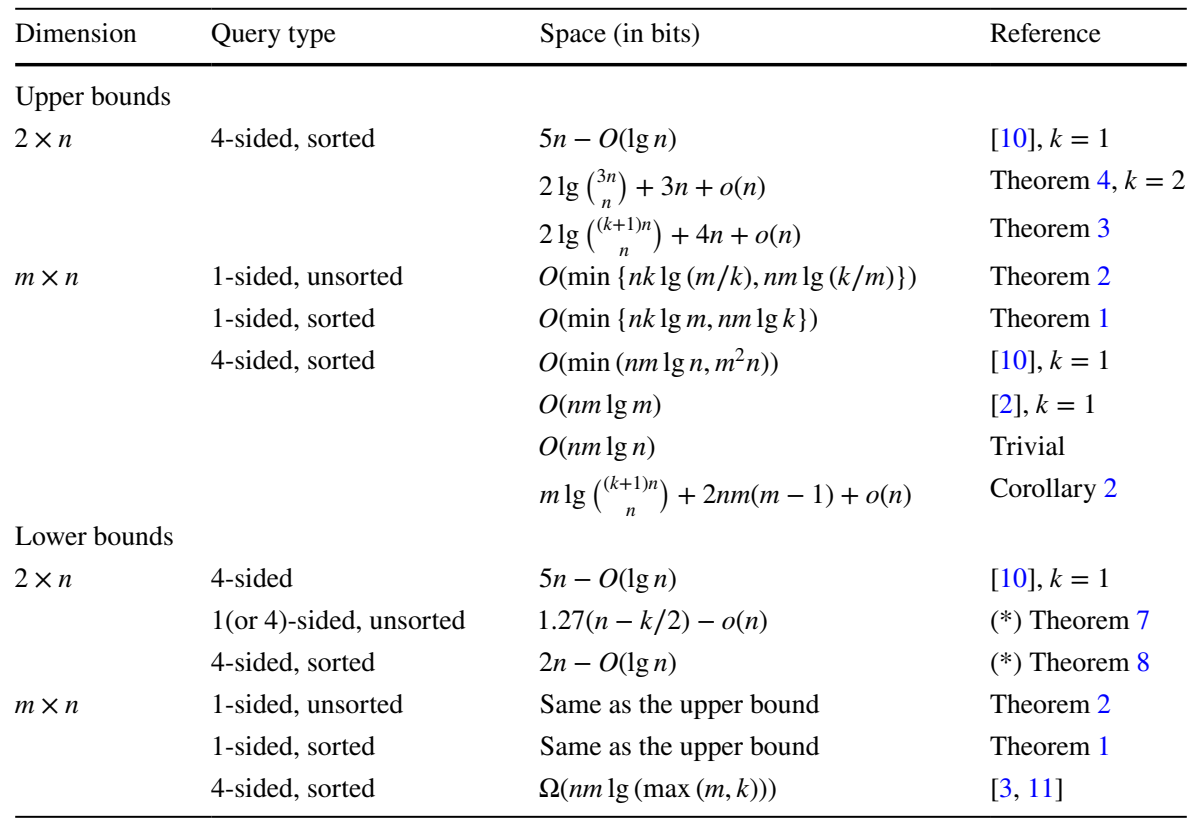

The lower bound results marked (*) (of Theorem 7 and 8) are for the additional space (in bits) necessary, assuming that encodings of Top- $k$ queries for both rows are given. Results in [10] support $O(1)$-time queries using $o(n)$ extra bits

$O(n)$ bits of the lower bound), since any Top- $k$ encoding for the array $A$ also needs to support the Top- $k$ queries on the individual rows. All these results are summarized in Table 1.

\section{Encoding 1-Sided Range Top-k Queries on Two Dimensional Array}

In this section, we consider the encoding of sorted and unsorted 1-sided Top- $k$ queries on a $2 \mathrm{D}$ array $A[1 \ldots m][1 \ldots n]$. In the rest of the paper, we use $(i, j)$ to denote the position in the $i$-th row and $j$-th column of a $2 \mathrm{D}$ array.

\subsection{Sorted 1-sided queries}

We first introduce an encoding by simply extending the encoding of sorted 1 -sided Top- $k$ queries for 1D array proposed by Grossi et al. [12]. Next we propose an optimal encoding for sorted 1-sided Top- $k$ queries on $A$. For a $1 \mathrm{D}$ array $A^{\prime}[1 \ldots n]$, define another $1 \mathrm{D}$ array $X[1 \ldots n]$ as follows. For $1 \leq i \leq k$, define $X[i]=i$. For $k<i \leq n, X[i]=X\left[i^{\prime}\right]$ if there exists a position $i^{\prime}$ which satisfies $\operatorname{Top}-k\left(1, i, A^{\prime}\right)=\operatorname{Top}-k(1, i-1, A) \backslash\left\{i^{\prime}\right\} \cup\{i\}$, and $X[i]=k+1$ otherwise. 
Then one can answer the Top- $k\left(1, i, A^{\prime}\right)$ by finding the rightmost occurrence of every element $1 \ldots k$ in $X[1 \ldots i]$. By representing $X$ (along with some additional auxiliary structures) using $n \lg k+O(n)$ bits, Grossi et al. [12] obtained an encoding which supports 1 -sided Top- $k$ queries on $A^{\prime}$ in $O(k)$ time. For a $2 \mathrm{D}$ array $A$, one can encode $A$ to support sorted 1-sided Top- $k$ queries by writing down the values of $A$ in column-major order into a $1 \mathrm{D}$ array, and using the encoding described above - resulting in the following encoding.

Proposition $1 A 2 D$ array $A[1 \ldots m][1 \ldots n]$ can be encoded using $m n \lg k+O(n)$ bits to support sorted 1-sided Top-k queries in $O(k)$ time.

Now we describe an optimal encoding of $A$ which supports sorted 1-sided Top- $k$ queries. For $1 \mathrm{D}$ array $A^{\prime}[1 \ldots n]$, we can define another $1 \mathrm{D}$ array $B^{\prime}[1 \ldots n]$ such that for $1 \leq i \leq n, B^{\prime}[i]=l$ if $A^{\prime}[i]$ is the $l$-th largest element in $A^{\prime}[1 \ldots i]$ with $l \leq k$, and $B^{\prime}[i]=k+1$ otherwise. Then we answer the Top- $k\left(1, i, A^{\prime}\right)$ query as follows. We first find the rightmost position $p_{1} \leq i$ such that $B^{\prime}\left[p_{1}\right] \leq k$. Then we find the rest of $k-1$ positions $p_{k}<p_{k-1}<\cdots<p_{2}$ such that for $2 \leq j \leq k, p_{j}$ is the rightmost position in $A^{\prime}\left[1 \ldots p_{j-1}-1\right]$ with $B^{\prime}\left[p_{j}\right] \leq k-j+1$. Finally, we return the positions $p_{1}, p_{2}, \ldots, p_{k}$. Therefore by storing $B^{\prime}$ using $n\lceil\lg (k+1)\rceil$ bits, we can answer the sorted 1-sided Top- $k$ queries on $A^{\prime}$. Also we can sort $A^{\prime}\left[p_{1}\right], \ldots, A^{\prime}\left[p_{k}\right]$ using the property that for $1 \leq b<a \leq k, A^{\prime}\left[p_{a}\right]<A^{\prime}\left[p_{b}\right]$ if and only if one of the following two conditions hold: (i) $B^{\prime}\left[p_{a}\right] \geq B^{\prime}\left[p_{b}\right]$, or (ii) $B^{\prime}\left[p_{a}\right]<B^{\prime}\left[p_{b}\right]$ and there exist at least $q=B^{\prime}\left[p_{b}\right]-B^{\prime}\left[p_{a}\right]$ positions $j_{1}, j_{2}, \ldots, j_{q}$ between $p_{a}$ and $p_{b}$ where $B^{\prime}\left[j_{r}\right] \leq B^{\prime}\left[p_{a}\right]$ for all $1 \leq r \leq q$.

We can extend this encoding for the sorted 1 -sided Top- $k$ queries on a $2 \mathrm{D}$ array $A$, to obtain an optimal encoding as stated in the following theorem.

Theorem 1 Given a $2 D$ array $A[1 \ldots m][1 \ldots n]$, there is an encoding of size $O(\min \{n m \lg k, n k \lg m\})$ bits, which can answer sorted 1-sided Top-k queries. Also, the space bound is asymptotically optimum.

Proof For $1 \leq j \leq n$, we first define the elements of $j$-th column in $A$ as $a_{1 j} \ldots a_{m j}$. Then we define the sequence $S_{j}=s_{1 j} \ldots s_{m j}$ such that for $1 \leq i \leq m, s_{i j}=l$ if $a_{i j}$ is the $l$-th largest element in $A[1 \ldots m][1 \ldots j]$ with $l \leq k$, and $s_{i j}=k+1$ otherwise. Since there exist $T=\sum_{p=0}^{\min (m, k)}\left(\begin{array}{l}m \\ p\end{array}\right)\left(\begin{array}{l}k \\ p\end{array}\right) p$ ! possible $S_{j}$ sequences ( $T$ is the total number of ways in which we can choose $p$ out of the $m$ rows for new entries into the Top- $k$ positions, summed over all possible values of $p$ ), we can store $S^{A}=S_{1} \ldots S_{n}$ using $n\lceil\lg T\rceil=O(\min \{n m \lg k, n k \lg m\})$ bits and we can answer the sorted 1-sided Top- $k(1, m, 1, j)$ queries on $A$ by the following procedure.

1. Find the rightmost column $q$, for some $q \leq j$, such that $S_{q}$ has $\ell>0$ elements $s_{p_{1} q}, \ldots, s_{p_{\ell} q}$ where $s_{p_{1} q}<\cdots<s_{p_{\ell} q}<k+1$. If $\ell=k$, we return the positions of $\left(p_{1}, q\right) \ldots\left(p_{k}, q\right)$ as the answers of the query, and stop. Otherwise (if $\left.\ell<k\right)$, we return the positions of $\left(p_{1}, q\right) \ldots\left(p_{\ell}, q\right)$, and

2. Repeat Step 1 by setting $k$ to $k-\ell$, and $j$ to $q-1$. 
We can return the positions in the sorted order of their corresponding values by applying the procedure to sort the answers of 1 -sided Top- $k$ queries on $S^{A}$, described above. This shows the upper bound.

We now show that $n \lg T$ bits are necessary to encode sorted 1-sided Top- $k$ queries on $A$. Suppose there are two distinct sequences $S^{A}=S_{1} \ldots S_{i}$ and $S^{A^{\prime}}=S_{1}^{\prime} \ldots S_{i}^{\prime}$ which give sorted 1-sided Top- $k$ encodings of $2 \mathrm{D}$ arrays $A$ and $A^{\prime}$, respectively. For $1 \leq b \leq n$, if $S_{b} \neq S_{b}^{\prime}$ then Top- $k(1, m, 1, b, A) \neq \operatorname{Top}-k\left(1, m, 1, b, A^{\prime}\right)$ by the definition of $S^{A}$ and $S^{A^{\prime}}$. Since for an $m \times n$ array, there are $T^{n}$ distinct sequences $S^{A_{1}} \ldots S^{A_{T^{n}}}$, We now prove for $1 \leq q \leq T^{n}$, each $S^{A_{q}}=S_{1}^{q} \ldots S_{n}^{q}$ has an array $A$ such that $S^{A}=S^{A_{q}}$, which completes the proof of the theorem.

Without loss of generality, suppose that all elements in $A$ come from the set $L=\{1, \ldots, m n\}$. Then we can reconstruct $A$ from the rightmost column using $S^{A_{q}}$ as follows. If $s_{j n}^{q} \leq k$, for $1 \leq j \leq m$, we assign the $s_{j n}^{q}$-th largest element in $L$ to $A[j][n]$. After we assign all values in the rightmost column with $s_{j n}^{q} \leq k$, we discard all assigned values from $L$, move to $(n-1)$-th column and repeat the procedure. After we assign all values in $A$ whose corresponding values in $S^{A_{q}}$ are smaller than $k+1$, we assign the remaining values in $L$ to remaining positions in $A_{q}$ which are not assigned yet. Thus for any $1 \leq b \leq n$, if $S_{b}^{q}$ has $\ell>0$ elements $s_{p_{1} b}, \ldots, s_{p_{\ell} b}$ where $s_{p_{1} b}<\cdots<s_{p_{\ell} b}<k+1$, then the $b$-th column in $A$ contains $\ell$-largest elements in $A[1 \ldots m][1 \ldots b]$ by the above procedure. This shows that $S^{A}=S^{A_{q}}$.

Note that this encoding takes less space than the encoding in the Proposition 1.

\subsection{Unsorted 1-sided queries}

In this section, we consider the encoding of unsorted 1 -sided Top- $k(1, m, 1, b)$ queries on a $2 \mathrm{D}$ array $A$. Let unsorted Top- $k(1, m, 1, b)=b_{1}, \ldots, b_{k}$ be the $k$ positions, ordered in their lexicographic order. Grossi et al [12] show that for any array $B[1 \ldots n]$ of size $n$, one can support unsorted Top- $k(1, i, B)$ queries for $1 \leq i \leq n$ using $n \lg k+O(n))$ bits with $O(k)$ query time. This implies that we can answer unsorted 1-sided Top- $k$ queries on $A$ using $m n \lg k+O(m n)$ bits with $O(k)$ query time, by converting the unsorted 1 -sided Top- $k$ queries on $A$ into the unsorted Top- $k$ queries on a 1D array of size $m n$ which is obtained by the values of $A$ in columnmajor order. Now we consider the another encoding which supports unsorted query using optimal space, if query time is not of concern, and show the following.

Theorem 2 Given a $2 D$ array $A[1 \ldots m][1 \ldots n]$, there is a data structure of size $O(\min \{n k \lg (m / k), n m \lg (k / m)\})$ bits which supports unsorted 1-sided Top- $k$ queries. Moreover, the space bound is asymptotically optimal.

Proof We first show the upper bound by considering the two cases, (i) $k \leq m$, and (ii) $k>m$ separately.

Case (i) $k \leq m$. In this case, we first encode the answers of unsorted Top- $k(1, m, 1,1)$ query using $\left\lceil\lg \left(\begin{array}{c}m \\ k\end{array}\right)\right\rceil$ bits. For $b>1$, the answers of Top- $k(1, m, 1, b)$ are the positions of $k$ largest values in $A$ out of $m+k$ positions, corresponding to the 
positions in Top- $k(1, m, 1, b-1)$ along with the positions $\{(1, b), \ldots,(m, b)\}$. Thus, for any $1<b \leq n$, if we know the answers of unsorted Top- $k(1, m, 1, b-1)$, we can encode the answers of unsorted Top- $k(1, m, 1, b)$ using $\left\lceil\lg \left(\begin{array}{c}k+m \\ k\end{array}\right)\right\rceil$ bits. Hence, the total space for encoding the answers for all the $n$ columns is $\left\lceil\lg \left(\begin{array}{c}m \\ k\end{array}\right)\right\rceil+(n-1)\left\lceil\lg \left(\begin{array}{c}k+m \\ k\end{array}\right)\right\rceil$ bits. We can find the answers of a 1 -sided Top- $k(1, m, 1, b)$ query as follows. We first decode the answers of Top- $k(1, m, 1,1)$, and decode the answers of unsorted 1-sided query from left to right until we decode the answers of $b$-th unsorted 1-sided Top- $k$ query.

Case (ii) $k>m$. When $1 \leq b \leq\lfloor k / m\rfloor$, it is obvious that $\operatorname{Top}-k(1, m, 1, b)=\{(i, j) \mid 1 \leq i \leq m, 1 \leq j \leq b\}$, i.e., all the positions within the query range are part of the answer. Therefore no extra space is needed for storing the answers of unsorted Top- $k(1, m, 1, b)$ queries for $1 \leq b \leq\lfloor k / m\rfloor$. When $b>\lfloor k / m\rfloor$, we can encode the answers of Top- $k(1, m, 1, b)$ queries using $(n-1-\lfloor k / m\rfloor)\left\lceil\lg \left(\begin{array}{c}k+m \\ k\end{array}\right)\right\rceil+\left\lceil\lg \left(\begin{array}{c}m \\ k-m\lfloor k / m\rfloor\end{array}\right)\right\rceil$ bits by using the similar encoding as described above (using $\left\lceil\lg \left(\begin{array}{c}m \\ k-m\lfloor k / m\rfloor\end{array}\right)\right\rceil$ bits for answering Top- $k(1, m, 1,\lfloor k / m\rfloor+1)$ query and $(n-1-\lfloor k / m\rfloor)\left\lceil\lg \left(\begin{array}{c}k+m \\ k\end{array}\right)\right\rceil$ bits for answering Top- $k(1, m, 1, b)$ queries for any $b>\lfloor k / m\rfloor+1)$. Also, we can find the answers of unsorted 1-sided Top- $k(1, m, 1, b)$ by a similar procedure as in Case (i). The only difference is when $1 \leq b \leq\lfloor k / m\rfloor$, we just report all positions in the sub-array $A[1 \ldots m][1 \ldots b]$.

From the above two cases, it follows that we can answer unosorted 1-sided Top- $k$ queries on $A$ using at most $n \log \left(\begin{array}{c}k+m \\ k\end{array}\right)$ bits. If $k<m$, then $n \log \left(\begin{array}{c}k+m \\ k\end{array}\right) \approx n k \log (m / k)$; and if $k>m$, then $n \log \left(\begin{array}{c}k+m \\ k\end{array}\right) \approx n m \log (k / m)$. Thus the space bound can be written as $O(\min \{n k \lg (m / k), n m \lg (k / m)\})$ bits. This shows the upper bound stated in the theorem.

Now we show the lower bound. Without loss of generality, suppose that all elements in the array come from the set $L_{n}=\{1, \ldots, m n\}$, and $k \leq m$ (we can prove the case when $k>m$ in a similar way). Then it is enough to show that there are $N=\left(\begin{array}{c}m \\ k\end{array}\right)\left(\begin{array}{c}k+m \\ k\end{array}\right)^{(n-1)}$ arrays $A_{1} \ldots A_{N}$ of size $m \times n$ such that for any $1 \leq s \neq t \leq N$, there exists $1 \leq b \leq n$ such that unsorted Top- $k\left(1, m, 1, b, A_{s}\right) \neq \operatorname{Top}-k\left(1, m, 1, b, A_{t}\right)$.

We prove the above statement by induction on $n$. When $n=1$, we assign $\{m, \ldots m-k+1\}$ to the answers of unsorted Top- $k(1, m, 1,1)$, and assign $L_{1}-\{m, \ldots m-k+1\}$ to remaining positions arbitrary. Since any $k$ positions in $(i, 1), 1 \leq i \leq m$ can be the answer of Top- $k(1, m, 1,1)$ query, there are $\left(\begin{array}{l}m \\ k\end{array}\right)$ arrays such that any two of them have different answers for the Top- $k(1, m, 1,1)$ query.

Now assume the inductive hypothesis that the above statement holds for some $n^{\prime}$ where $1 \leq n^{\prime}<n$, and that there are $N^{\prime}=\left(\begin{array}{c}m \\ k\end{array}\right)\left(\begin{array}{c}k+m \\ k\end{array}\right){ }^{\left(n^{\prime}-1\right)}$ arrays $A_{1} \ldots A_{N^{\prime}}$, satisfying the above statement. Let a set $M=L_{\left(n^{\prime}+1\right)}-L_{n^{\prime}}=\left\{m\left(n^{\prime}+1\right), \ldots m\left(n^{\prime}+1\right)-k+1\right\}$ and $M^{\prime}=\emptyset$.

To prove the inductive step for $n^{\prime}+1$, we first pick an arbitrary $A_{s}, 1 \leq s \leq N^{\prime}$ from the set of arrays $\left\{A_{1} \ldots A_{N^{\prime}}\right\}$ and add the $\left(n^{\prime}+1\right)$-th column to $A_{s}$ (none of the positions in this column have an assigned value). Then for some integer $\alpha$ where $0 \leq \alpha \leq k$, we pick the answers of unsorted Top- $k\left(1, m, 1, n^{\prime}+1\right)$ query by choosing $\alpha$ positions from the set Top- $k\left(1, m, 1, n^{\prime}, A_{s}\right)=\left\{n_{1}^{\prime} \ldots n_{k}^{\prime}\right\}$ and choosing the remaining $k-\alpha$ positions from the $\left(n^{\prime}+1\right)$-th column. We then choose $\alpha$ elements from $M$ and add them to the $\alpha$ positions chosen from the set $\left\{n_{1}^{\prime} \ldots n_{k}^{\prime}\right\}$. 
When we assign a value $x \in M$ to $(i, j) \in\left\{n_{1}^{\prime} \ldots n_{k}^{\prime}\right\}$, we delete $x$ from $M$, set $M^{\prime}=M^{\prime} \cup A_{s}[i][j]$ and $A_{s}[i][j]=x$. Since $n_{i}^{\prime} \leq n^{\prime} m$ for all $1 \leq i \leq k$, it is easy to show that this does not change the answers of unsorted Top- $k\left(1, m, 1, b, A_{s}\right)$ for all $1 \leq b \leq n^{\prime}$. Next, we assign the remaining values in $M$ to the $k-\alpha$ chosen positions in the $\left(n^{\prime}+1\right)$-th column, and finally assign the values in $M^{\prime}$ to the remaining positions in that column arbitrarily. Since there are $\sum_{\alpha=1}^{k}\left(\begin{array}{c}k \\ \alpha\end{array}\right)\left(\begin{array}{c}m \\ k-\alpha\end{array}\right)=\left(\begin{array}{c}m+k \\ k\end{array}\right)$ ways to select the answers of unsorted Top- $k\left(1, m, 1, n^{\prime}+1\right)$ query, and for each $s$, there are $Q=\left(\begin{array}{c}m+k \\ k\end{array}\right)$ arrays $A_{s 1} \ldots A_{s Q}$ such that for all $1 \leq t \neq \ell \leq Q$ and $1 \leq b \leq n^{\prime}$, unsorted $\operatorname{Top}-k\left(1, m, 1, b, A_{s t}\right)=\operatorname{Top}-k\left(1, m, 1, b, A_{s \ell}\right)=\operatorname{Top}-k\left(1, m, 1, b, A_{s}\right)$ but unsorted Top- $k\left(1, m, 1, n^{\prime}+1, A_{s t}\right) \neq \operatorname{Top}-k\left(1, m, 1, n^{\prime}+1, A_{s \ell}\right)$. Therefore the above statement holds for $n^{\prime}+1$ whenever it holds for $n^{\prime}$, which proves the theorem.

Note that we can construct the encoding of Theorem 2 in $O(m n \lg k+n k)$ time by maintaining a min-heap of size at most $k$. More precisely, we insert the values of $A$ in column-major order and delete the minimum value in the heap when the size of the heap is more than $k$. We can answer the position of the $k$ largest values in $A[1, m]$ $[1, i]$ for $1 \leq i \leq n$, by scanning the heap after every $m$-th insertion, in $O(k)$ time.

Remark Let $S_{\text {sorted }}$ and $S_{\text {unsorted }}$ be the space needed to encode the sorted and unsorted 1-sided Top- $k$ queries respectively. For 1D array, Gawrychowski and Nicholson showed that $S_{\text {sorted }} / S_{\text {unsorted }} \leq 2$ (thus, the space requirements are asymptotically same for both case) [8]. In contrast, in 2D array case when $k=m$, $S_{\text {sorted }} / S_{\text {unsorted }} \leq \lg k$ by Theorems 1 and 2, which implies the gap between the space needed to encode the 1-sided Top- $k$ queries for sorted and unsorted case for a $2 \mathrm{D}$ array is significantly more than the case for a $1 \mathrm{D}$ array.

\section{Encoding 4-Sided Top-k Queries on on $2 \times n$ Array}

In this section, we give an encoding which supports general Top- $k$ queries on $m \times n$ $2 \mathrm{D}$ array $A$. Note that if query time is not of concern in the above data structure, one can simply consider the $m n\lceil\lg (m n)$-bit trivial encoding for answering Top- $k$ queries on $A$, by storing the rank (i.e., the position in sorted order) of all the elements in $A$. We first introduce an $O(m n \lg n)$-bit data structure which supports Top- $k$ query in $O(k)$ time by using the RMQ encoding of Brodal et al. [2].

Proposition 2 Given a $2 D$ array $A[1 \ldots m][1 \ldots n]$, there exists an $O(m n \lg n)$-bit data structure to support unsorted Top- $k(i, j, a, b, A)$ in $O(k)$ time for $1 \leq a, b \leq m$ and $1 \leq i, j \leq n$.

Proof We use a data structure similar to the one outlined in [4] (based on Frederikson's heap selection algorithm [7]) for answering unsorted Top- $k$ queries in 1D 
array $^{3}$. First encode $A$ using $O(m n \lg n)$ bits to support RMQ (range maximum) queries in constant time for any rectangular range in $A$. This encoding also supports finding the rank of any element in $A$ in $O(1)$ time [3]. Next, let $x=A\left[x_{1}\right]\left[x_{2}\right]$ be the maximum value in $A[i \ldots j][a \ldots b]$, which can be found using an RMQ query on $A$. Then consider the 4-ary heap obtained by the following procedure. The root of the heap is $x$, and its four subtrees are formed by recursively constructing the 4-ary heap on the sub-arrays $A\left[i \ldots x_{1}-1\right][a \ldots b], A\left[x_{1}+1 \ldots j\right][a \ldots b], A\left[x_{1}\right]\left[a \ldots x_{2}-1\right]$ and $A\left[x_{1}\right]\left[x_{2}+1 \ldots b\right]$, respectively. Now, we can find the $k$ largest elements in the above 4-ary heap in $O(k)$ time using the algorithm proposed by Frederickson [7] (note that this algorithm only builds a heap with $O(k)$ nodes which is a connected subgraph of the above 4-ary heap).

We now introduce alternative encoding to support Top- $k$ queries on an $m \times n 2 \mathrm{D}$ array $A$, which take less space than the trivial encoding for small $m$. The overall idea is as follows. We first show that $4 n$ bits are sufficient for answering sorted 4-sided Top- $k$ queries on $A$ for $m=2$ when encodings for answering sorted 2-sided Top- $k$ queries for each row are given. This encoding is obtained by encoding the binary DAG which is defined later. After that, we extend the encoding into $m \times n$ array and obtain $\left(m \lg \left(\begin{array}{c}(k+1) n \\ n\end{array}\right)+2 n m(m-1)+o(n)\right)$-bit encoding for answering Top- $k$ queries on $A$. Note that this encoding takes less space than the trivial $m n\lceil\lg (m n)\rceil$ bit encoding when $m=o(\lg n)$.

Now we describe the encoding of sorted 4-sided Top- $k$ on $A$ when $m=2$. For $1 \leq i \leq 2$, let $A_{i}[1 \ldots n]$ be the array of the $i$-th row in $A$, and assume that sorted 2-sided Top- $k$ encodings on $A_{1}$ and $A_{2}$ are already given. When $k=1$, i.e., to answer RMQ queries on $A$, one can use the joint Cartesian tree of Golin et al. [10]. The joint Cartesian tree of constructs a conceptual binary tree analogous to a Cartesian tree, storing a bit indicating which row the maximum element in the range comes from, splitting the range corresponding to the node at the position of the maximum element, and then recursing on each subrange. Thus by storing an extra $n$ bits (one at each node of the conceptual binary tree), they showed that one can answer $\mathrm{RMQ}(1,2, a, b, A)$ queries for any $1 \leq a, b \leq n$, if the encodings for answering RMQ on $A_{1}$ and $A_{2}$ are given. See [10] for details. To answer the sorted 4-sided Top- $k$ queries with $k \geq 2$, we extend the idea of a joint Cartesian tree into a DAG-based structure, denoted by $D_{A}^{k}$, which is defined as follows.

Every node $p$ in $D_{A}^{k}$ is labeled with some closed interval $I_{p}=[a, b]$, where $1 \leq a, b \leq n$. In this case, we use both Top- $k(p)$ and Top- $k\left(I_{p}\right)$ to refer to the sorted Top- $k(1,2, a, b, A)$ query. For a node $p$ with label $I_{p}=[a, b]$ in $D_{A}^{k}$ and $1 \leq i \leq k$, let $\left(p_{r}^{i}, p_{c}^{i}\right)$ be the position of the $i$-th largest element in $A[1,2][a \ldots b]$. Now we define $D_{A}^{k}$ as follows (see Fig. 1 for an example.).

1. The root of $D_{A}^{k}$ is labeled with the range $[1, n]$.

\footnotetext{
${ }^{3}$ Brodal et al. [4] also give another structure to answer sorted Top- $k$ queries, with the same time and space bounds.
} 


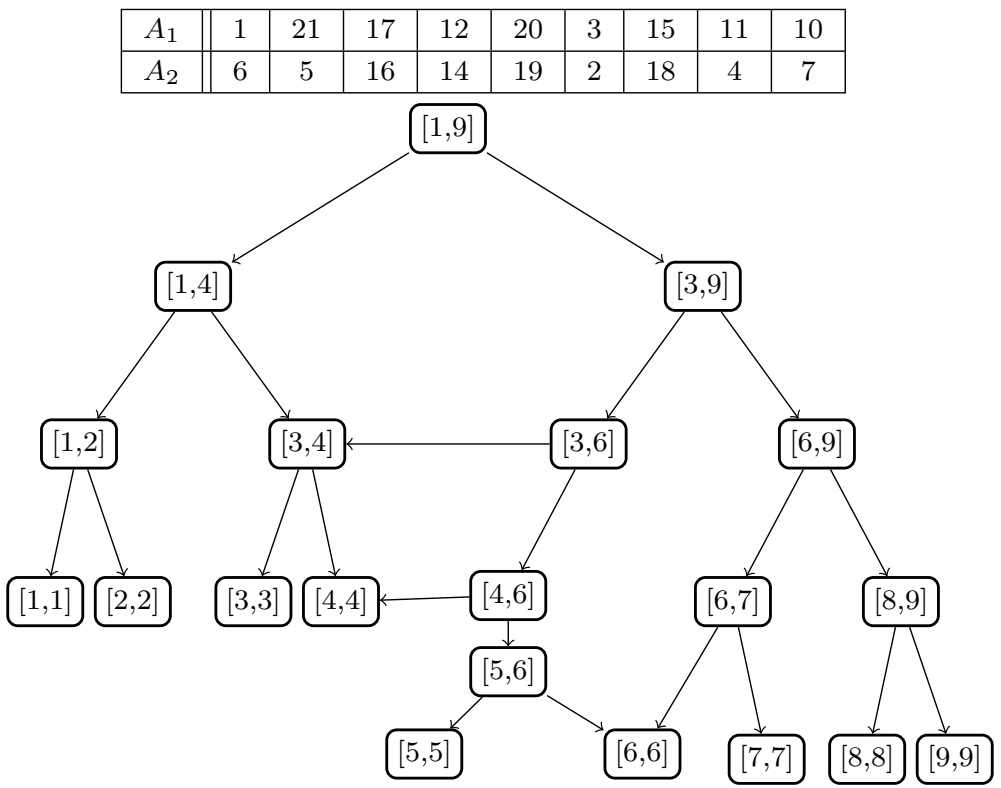

Fig. $12 \times n$ array $A$ and the DAG $D_{A}^{3}$

2. A node $p$ with label $I_{p}=[a, b]$ does not have any child node (i.e., leaf node) if $2(b-a+1) \leq k$.

3. Suppose there exists a non-leaf node $p$ with label $I_{p}=[a, b]$ in $D_{A}^{k}$, and let $a^{\prime}$ and $b^{\prime}\left(a \leq a^{\prime} \leq b^{\prime} \leq b\right)$ be the leftmost and rightmost column indices among the answers of Top- $k(p)$, respectively. If $a<b^{\prime}$, then the node $p$ has a node with label $\left[a, b^{\prime}-1\right]$ as a left child. Similarly, if $a^{\prime}<b$, the node $p$ has a node with label $\left[a^{\prime}+1, b\right]$ as a right child.

The following lemma states some useful properties of $D_{A}^{k}$.

Lemma 1 Let $A$ be a $2 \times n$ array. The following statements hold.

(i) For any two distinct nodes $p$ and $q$ in $D_{A}^{k}$, Top- $k(p) \neq$ Top- $k(q)$ (i.e., any two distinct nodes have different Top- $k$ answers).

(ii) $I_{p} \subset I_{q}$ if and only if $p$ is descendant of $q$ in $D_{A}^{k}$.

(iii) For any interval $[a, b]$ with $1 \leq a \leq b \leq n$, there exists a unique node $p$ in $D_{A}^{k}$ which satisfies $(i)[a, b] \subset I_{p}$, and (ii) label of any descendant of $p$ does not contain $[a, b]$. Furthermore, for such a node $p$, Top- $k([a, b])=\operatorname{Top}-k(p)$.

\section{Proof}

(i) From the construction of $D_{A}^{k}$, one can observe that if there is a node with label $[a, b]$ in $D_{A}^{k}$, with $1<a \leq b<n$, then both $(a-1)$-th and $(b+1)$-th column contain at least one element that is larger than the ele- 
ments in Top- $k([a, b])$, which implies Top- $k([a, b]) \neq \operatorname{Top}-k([a, b+1])$ and Top- $k([a-1, b]) \neq \operatorname{Top}-k([a, b])$. Now suppose that there are two distinct nodes with labels $[a, b]$ and $\left[a^{\prime}, b^{\prime}\right]$ with $b<b^{\prime}$ such that $\operatorname{Top}-k([a, b])=\operatorname{Top}-k\left(\left[a^{\prime}, b^{\prime}\right]\right)$, then Top- $k([a, b+1])=\operatorname{Top}-k\left(\left[a^{\prime}, b^{\prime}\right]\right)$, contradicting the fact that Top- $k([a, b]) \neq \operatorname{Top}-k([a, b+1])$. The case when $b>b^{\prime}$, $a>a^{\prime}$ or $a<a^{\prime}$ is analogous.

(ii) Let $I_{p}=\left[a_{p}, b_{p}\right]$ and $I_{q}=\left[a_{q}, b_{q}\right]$. From the construction of $D_{A}^{k}$, it is the case that if $p$ is a descendant of $q$, then $I_{p} \subset I_{q}$. Now, suppose that there are two nodes $p, q \in D_{A}^{k}$ such that $I_{p} \subset I_{q}$ but $p$ is not descendant of $q$. Then there exists a node $q^{\prime}$ which satisfies (i) $q^{\prime}$ is a descendant of $q$, (ii) $I_{p} \subset I_{q^{\prime}}$, and (iii) no child of $q^{\prime}$ whose label contains $I_{p}$. Since neither of labels of the children of $q^{\prime}$ contain $I_{p}$, all the positions of Top- $k\left(q^{\prime}\right)$ are between $a_{p}$-th and $b_{p}$-th column. (otherwise, there always exists a child $q^{\prime \prime}$ of $q^{\prime}$ which satisfies $I_{q^{\prime \prime}} \subset I_{p}$ ). But this would imply that Top- $k\left(q^{\prime}\right)=\operatorname{Top}-k(p)$, which leads to a contradiction with Lemma 1(i).

(iii) We first show that there exists a unique node $p$ in $D_{A}^{k}$ such that $I_{p}$ contains the interval $[a, b]$ and none of labels of the children of $p$ contain $[a, b]$. We then show that the Top- $k(p)=\operatorname{Top}-k([a, b])$.

Since label of the root in $D_{A}^{k}$ contains all column indices in $A$, it is easy to see that there exists at least one node $p$ with label $I_{p}=\left[a_{p}, b_{p}\right]$ in $D_{A}^{k}$ such that $[a, b] \subset I_{p}$ but no child of $I_{p}$ contains $[a, b]$. Suppose that there exists another node $p^{\prime}$ with label $I_{p^{\prime}}=\left[a_{p}^{\prime}, b_{p}^{\prime}\right]$ in $D_{A}^{k}$ such that $[a, b] \subset I_{p^{\prime}}$ but there is no child of $p^{\prime}$ whose label contains $[a, b]$. By Lemma 1(ii), it follows that $I_{p} \not \subset I_{p^{\prime}}$ and $I_{p^{\prime}} \not \subset I_{p}$ (otherwise, one of them would be a descendant of the other, contradicting the conditions on $p$ and $\left.p^{\prime}\right)$. Now, suppose that $a_{p}<a_{p}^{\prime}<b_{p}<b_{p}^{\prime}$ (the case when $a_{p}^{\prime}<a_{p}<b_{p}^{\prime}<b_{p}$ is analogous). Then there exists a column $c<a_{p}^{\prime}$ such that $p$ has a child node with label $\left[c, b_{p}\right]$ where $[a, b] \subset\left[c, b_{p}\right]$ by the property of $D_{A}^{k}$ (note that $a_{p}^{\prime} \leq a \leq b \leq b_{p}$ ), contradicting the fact that $p$ does not have such a child. This shows that there is a unique such $p$ in $D_{A}^{k}$.

Now we claim that Top- $k([a, b])=\operatorname{Top}-k(p)$. Suppose that there exist a $c \notin[a, b]$ in $I_{p}$ such that column $c$ contains at least one of the answers to Top- $k(p)$. Also without loss of generality, we assume that $c<a$ (the case when $c>b$ can be handled in a similar way). Then by the property of $D_{A}^{k}, p$ has a child with label $\left[c+1, b_{p}\right]$ which still contains $[a, b]$, contradicting the fact that $p$ does not have such a child.

By Lemma 1(iii), if the DAG $D_{A}^{k}$ and the answers for each sorted 2-sided Top- $k$ queries corresponding to all the nodes in $D_{A}^{k}$ are given, then we can answer any sorted Top- $k(1,2, a, b, A)$ query by finding the corresponding node in $p$ in $D_{A}^{k}$ which satisfies Top- $k(1,2, a, b, A)=\operatorname{Top}-k(p)$.

We now describe how to encode $D_{A}^{k}$ using at most $4 n$ bits. The main idea of our encoding is as follows. For each node in $D_{A}^{k}$, we assign at most 2 bits (except the root node, which is assigned $k$ bits) while traversing all the nodes in the level order. These bits enable us to answer Top- $k$ queries on the range corresponding to each node in $D_{A}^{k}$, using the Top- $k$ answers of the nodes in the previous level (more specifically, one of the parent nodes), and the Top- $k$ encodings of the individual rows. 
1. Mark the root of $D_{A}^{k}$ as visited, and add its children into visit-list, which is an ordered list such that for two nodes $p$ and $q$ in visit-list, $p$ comes before $q$ in visitlist if and only if $l(p)<l(q)$ or $l(p)=l(q)$ and $p$ precedes $q$ in the DAG $(l(p)$ denotes the level of the node $p$ which is defined as the number of edges in the longest path from root to $p$ in $D_{A}^{k}$ ).

2. Find the leftmost unvisited or half-visited node $p$ from visit-list which satisfies one of the following conditions (without loss of generality, assume that $x \leq y$ ).

(a) Number of first or second candidates of $p$ is less than 2 .

(b) First or second candidates of $p$ are $(1, x)$ and $(2, y)$, and there exists no node $p^{\prime}$ in visit-list such that (a) $I_{p} \subset I_{p^{\prime}}$, or (b) $p^{\prime}$ precedes $p$ and $x \in I_{p^{\prime}}$, or (c) $I_{p}$ precedes $I_{p^{\prime}}$ and $y \in I_{p^{\prime}}$.

Then we continue the traversal from $p$.

3. Let $q$ be a parent of $p$. If (i) $\mid$ Top- $k(q)-$ Top- $k(p) \mid=1$, or (ii) $\mid$ Top- $k(q)-$ Top- $k(p) \mid=$ 2 and $p$ is half-visited, or (iii) the number of first or second candidates of $p$ is less than 2 , then mark $p$ as visited, delete $p$ from the visit-list, and insert $p$ 's children (if any) to visit-list. If none of these three conditions hold, then mark $p$ as half-visited.

4. Repeat Steps 2 and 3 until all the nodes in $D_{A}^{k}$ are marked as visited.

Fig. 2 Modified level-order traversal of $D_{A}^{k}$

By Lemma 1(iii), this encoding is enough to answer all possible Top- $k(1,2, a, b, A)$ queries for any $1 \leq a, b \leq n$. However, since there exists at most $O(k n)$ nodes in $D_{A}^{k}$ (see Lemma 2), this encoding takes $O(k n)$ bits. To make the space independent to $k$, we skip some redundant nodes in $D_{A}^{k}$ (i.e., nodes for which the answers of Top- $k$ on that nodes can be answered using the information obtained by some of the already traversed nodes, without any extra information). We modify the original level order to modified level order, which will be describe later, and show that if we encode $D_{A}^{k}$ according to the modified level order, we can encode $D_{A}^{k}$ at most $4 n$ bits, by skipping the redundant nodes during the traversal.

Modified level-order For two nodes $p_{i}$ with label $I_{p_{i}}=\left[a_{i}, b_{i}\right]$ and $p_{j}$ with label $I_{p_{j}}=\left[a_{j}, b_{j}\right]$ which satisfy $I_{p_{i}} \not \subset I_{p_{j}}$ and $I_{p_{j}} \not \subset I_{p_{i}}$, we say the node $p_{i}$ precedes the node $p_{j}$ if $a_{i}<a_{j}$. Now, let $q$ be one of the parents of the node $p$ with label $I_{p}=[a, b]$ (note that a node can have multiple parents in a DAG). Note that $1 \leq \mid$ Top- $k(q)-$ Top- $k(p) \mid \leq 2$, since $I_{p}$ contains all the answers of Top- $k(q)$ except one or both positions from the column $a-1$ or from the column $b+1$. Also let $f_{p}$ and $s_{p}$ be the number of positions in Top- $k(q) \cap$ Top- $k(p)$ on the first and the second row respectively. Now we consider the following two cases:

- Case 1 (|Top- $k(q)-\operatorname{Top}-k(p) \mid=1)$ : In this case, the positions of Top- $(k-1)(p)$ are already contained in the answers of Top- $k(q)$, and the $k$-th largest element in $A[1,2][a, \ldots, b]$ is either the $\left(f_{p}+1\right)$-th largest element in $A_{1}[a, \ldots, b]$ or the $\left(s_{p}+1\right)$-th largest element in $A_{2}[a, \ldots, b]$ (we call them as first-candidates at node $p$ ). 
- Case $2(\mid \operatorname{Top}-k(q)-$ Top- $k(p) \mid=2)$ : In this case, the positions of Top- $(k-2)(p)$ are already contained in the answers of Top- $k(q)$, and the $(k-1)$-th largest element in $A[1,2][a, \ldots, b]$ is the one of the the first-candidates at node $p$. Now suppose $(k-1)$-th largest element in $A[1,2][a, \ldots, b]$ is on the first row (the other case is analogous). Then again, the $k$-th largest element in $A[1,2][a, \ldots, b]$ is on the one of the positions of $\left(f_{p}+2\right)$-th largest element in $A_{1}[a, \ldots, b]$ and $\left(s_{p}+1\right)$ -th largest element in $A_{2}[a, \ldots, b]$ (we call them as second-candidates at node $p$ ).

Note that if $f_{p}$ and $s_{p}$ are given, the first and second-candidates at node $p$ can be found using the Top- $k$ encodings of $A_{1}$ and $A_{2}$. Figure 2 shows the overall procedure of modified level-order. While traversing the nodes of $D_{A}^{k}$ in the modified level order, we classify the nodes as visited, half-visited, or unvisited. All the nodes are initially unvisited, and the traversal continues until all the nodes in $D_{A}^{k}$ are visited. For example, we traverse the nodes of $D_{A}^{3}$ in Fig. 1 as: $[1,9] \rightarrow[1,4] \rightarrow[1,4] \rightarrow[3,9] \rightarrow[1,2] \rightarrow[1,2] \rightarrow[3,6] \rightarrow[6,9] \rightarrow[6,9] \rightarrow[1,1]$ $\rightarrow[8,9] \rightarrow[8,9] \rightarrow[3,3] \rightarrow[4,4] \rightarrow[5,6] \rightarrow[8,8] \rightarrow[9,9] \rightarrow[5,5] \rightarrow[6,6]$ (here each node is denoted as its label).

Picking the positions For a node $p=[a, b] \in D_{A}^{k}$, p picks the position $(x, y)$ if (i) $(x, y)$ is among the Top- $k$ positions of $p$, and (ii) this information (that $(x, y)$ is among the answers to Top- $k(p)$ query) does not follow from the Top- $k$ positions of any of the visited or half-visited before $p$ in the modified level-order. By storing the information of all picked positions at node $p$, we can answer Top- $k(p)$ by combining the answers of Top- $k$ positions of some visited or half-visited nodes before $p$.

When the traversal starts at the root node of $D_{A}^{k}$, the root node picks the positions of $k$-largest values among the answers of Top- $k\left(1, n, A_{1}\right)$ and Top- $k\left(1, n, A_{2}\right)$ queries, and theses positions can be indicated using $k$ bits, since we assume that Top- $k$ encodings of $A_{1}$ and $A_{2}$ are given.

Next, suppose we visit an unvisited non-root node $p$ where $|\operatorname{Top}-k(q)-\operatorname{Top}-k(p)|=1$, where $q$ is a parent of $p$ (note that $q$ is always visited before $p$ in modified level order). In this case, since we can answer the positions of $(k-1)$ largest elements in $A$ using Top- $k(q), p$ picks at most one position, which is among the first-candidates at $p$. Thus, we can store the picked position at node $p$ using one extra bit. The case when $|\operatorname{Top}-k(q)-\operatorname{Top}-k(p)|=2$ can be handled similarly, other than $p$ picks at most two positions (one from the first-candidates and another from the second-candidates), and this information can be stored using at most two extra bits. The following lemma shows that the size of $D_{A}^{k}$ is $O(k n)$, which in turn gives a simple $O(k n)$-bit space bound by storing the information of all the picked positions at each node of $D_{A}^{k}$.

Lemma 2 Given $2 \times n$ array $A$ and $D A G D_{A}^{k}$, there are at most $6 k n$ nodes in $D_{A}^{k}$.

Proof It is enough to show that there are at most $2 \mathrm{kn}$ non-leaf nodes in $D_{A}^{k}$. Let $P_{[\alpha, \beta]}=\left\{I_{p_{1}}=\left[a_{1}, b_{1}\right], I_{p_{2}}=\left[a_{2}, b_{2}\right] \ldots I_{p_{t}}=\left[a_{t}, b_{t}\right]\right\}$ be a set of labels of $t$ non-leaf nodes $p_{1}, p_{2}, \ldots, p_{t}$ in $D_{A}^{k}$ where all the nodes $p_{1}, p_{2}, \ldots, p_{t}$ picks $(\alpha, \beta)$.

Now we claim that $t$ is at most $k$. To prove a claim, suppose $t=k+1$. 
Then it is clear that for any $I_{p_{i}}, I_{p_{j}} \in P_{[\alpha, \beta]}, I_{p_{i}} \not \subset I_{p_{j}}$ and $I_{p_{j}} \not \subset I_{p_{i}}$ by the modified level-order traversal of $D_{A}^{k}$. Therefore without loss of generality, assume that $a_{1}<a_{2} \cdots<a_{k+1}<\beta<b_{1}<b_{2} \cdots<b_{k+1}$. Also we can easily show that for $1 \leq i<k+1$, there is a position at $\left(b_{i}+1\right)$-th column whose corresponding value is larger than $A\left[p_{i_{r}}^{k}\right]\left[p_{i_{c}}^{k}\right]$ by the construction algorithm of $D_{A}^{k}$. Therefore for $1 \leq i \leq k$, the node $A[1,2]\left[a_{k+1} \ldots b_{k+1}\right]$ has $k$ positions at $\left(b_{i}+1\right)$-th columns which have larger values than both $A[\alpha][\beta]$, contradicts to the fact that $(\alpha, \beta) \in \operatorname{Top}-k\left(p_{k+1}\right)$.

We now describe how to make the space usage of our encoding to be independent of $k$ - from $O(k n)$ to $O(n)$. Suppose there exists two non-root nodes $p$ and $q$ in $D_{A}^{k}$ where the first (or second) candidates of $p$ are contained in $q$, and the candidates of $p$ and $q$ are not distinct. In this case, the modified level order always visits $q$ prior to $p$, and gives a 'chance' not to pick any position at node $p$, although $q$ is not an ancestor of $p$. Using this property, we now prove the following lemma, which bounds the size of our encoding by showing that if we store the all picked positions according to the modified level order, we can encode $D_{A}^{k}$ in space independent to $k$.

Lemma 3 Given $2 \times n$ array $A[1,2][1 \ldots n]$ and $D A G D_{A}^{k}$, any position in $A$ is picked at most twice while we traverse all nodes in $D_{A}^{k}$ in the modified level order.

Proof Suppose that a position $(i, j)$ is among the answers of Top- $k$ query on the $t$ distinct nodes $p_{1}, p_{2}, \ldots, p_{t}$ where $I_{p_{i}}=\left[a_{i}, b_{i}\right]$ for $i \in\{1,2, \ldots, t\}$, but not among the answers of Top- $k$ query on their parent nodes. For $1 \leq a, b \leq t$ if $p_{a}$ is an ancestor of $p_{b}$, we don't pick $(i, j)$ at the node $p_{b}$ by the modified level order traversal algorithm (Note that $p_{a}$ is traversed before $p_{b}$ ). Therefore without loss of generality, we assume that for all $1 \leq a \neq b \leq t, I_{p_{a}} \not \subset I_{p_{b}}$ and $I_{p_{b}} \not \subset I_{p_{a}}$. Now we claim that for every position $(i, j)$ in $A$, at most two nodes from $p_{1}, \ldots, p_{t}$ pick $(i, j)$.

To prove the claim, for $1 \leq a<b<c \leq t$, suppose there exists three nodes $p_{a}, p_{b}$, and $p_{c}$ where all of these three nodes picks $(i, j)$, and let $\left(i^{\prime}, j^{\prime}\right)$ be the another (first or second)-candidate of $p_{b}$. Then by the modified level-order traversal algorithm we do not pick $(i, j)$ at $p_{b}$ (note that $j^{\prime} \in I_{p_{a}}$ or $j^{\prime} \in I_{p_{b}}$ ), which contradicts the assumption.

For example, during the traversal of $D_{A}^{3}$ in Fig. 1 according to modified level order, the position(s) picked at each node are: $\{(1,2),(1,5),(2,5)\} \rightarrow(1,3) \rightarrow(2,3) \rightarrow(2,7) \rightarrow(1,1) \rightarrow(1,2) \rightarrow \epsilon \rightarrow(1,7) \rightarrow(1,8) \rightarrow \epsilon \rightarrow \epsilon \rightarrow(2,4)$ $\rightarrow \epsilon \rightarrow(1,6) \rightarrow(1,9) \rightarrow(2,9) \rightarrow \epsilon \rightarrow \epsilon \rightarrow \epsilon \rightarrow \epsilon \rightarrow \epsilon \rightarrow \epsilon \rightarrow \epsilon$, respectively ( $\epsilon$ indicates that no position is picked). Now we prove our main theorem.

Theorem 3 Given a $2 \times n$ array $A$, if there exists an $S(n, k)$-bit encoding to answer sorted 2-sided Top- $k$ queries on a $1 D$ array of size $n$ in $T(n, k)$ time and such encoding can be constructed in $C(n, k)$ time, then we can construct an encoding of $A$ that uses $2 S(n, k)+4 n$ bits which can be constructed in $O\left(C(n, k)+k^{2} n^{2}+k n T(n, k)\right)$ time, for answering Top- $k$ queries on $A$. 
Proof For $1 \leq i \leq j \leq n$, we first use $2 S(n, k)$ bits to support sorted 2-sided $\operatorname{Top}-k(1,1, i, j, A)$ and Top- $k(2,2, i, j, A)$ queries. To answer Top- $k(1,2, i, j, A)$ queries, from Lemma 1(iii), we note that it is enough to encode the answers to the sorted Top- $k$ queries corresponding to all the nodes in $D_{A}^{k}$. We encode these answers into a bit string $X$ while traversing the DAG $D_{A}^{k}$ as follows. When the traversal begins at the root, $X$ is initialized to a $k$-bit string, which stores information for answering Top- $k(1,2,1, n, A)$ query (namely, the $i$-th bit stores 0 or 1 depending on whether the $i$-th largest element in the range comes from the top or bottom row, respectively). Now we traverse $D_{A}^{k}$ in the modified level order from the root node. Whenever we find a node $p$ in Step (2) of the traversal algorithm described above, and if we pick a position $(x, y)$ at node $p$, we append a single bit to $X$ to find the answer from $p$ 's first (or second) candidate.

Note that we can find such $p$ in $O(k n)$ time by the Lemma 2 and find the first (or second) candidates of node $p$ in $T(n, k)+O(1)$ time using the encoding of Top- $k$ queries on individual rows and pre-visited nodes other than root node, which takes $T(n, k)+O(k \lg k)$ time. Finally we can check whether one of the position in the first (or second candidates) of $p$ is picked at node $p$ or not in $O(n)$ time. Therefore whenever we traverse node $p, O(k n+T(n, k))$ time is sufficient for encoding a bit in $X$ to find the answer of Top- $k(p)$ query. Since we traverse any node at most twice in the modified level order, and since $D_{A}^{k}$ has at most $6 k n$ nodes by Lemma 2, we can construct the encoding in $O\left(C(n, k)+k^{2} n^{2}+k n T(n, k)\right)$ time in total. Also by Lemma 3 , $|X| \leq 4 n$ after we traverse all the nodes in $D_{A}^{k}$.

To decode answers of Top- $k$ queries corresponding to the nodes in $D_{A}^{k}$ from $X$, we first construct the root and its children from the first $k$ bits, and whenever we find a node $p$ with label $I_{p}=[a, b]$ in Step (2) of the traversal algorithm described above, we decode $\left(p_{r}^{k}, p_{c}^{k}\right)$ when $p$ is unvisited and $\mid \operatorname{Top}-k(q)-$ Top- $k(p) \mid=1$, or $p$ is halfvisited and $\mid$ Top- $k(q)-$ Top- $k(p) \mid=2$. Also we decode $\left(p_{r}^{k-1}, p_{c}^{k-1}\right)$ when $p$ is unvisited and $|\operatorname{Top}-k(q)-\operatorname{Top}-k(p)|=2$. The positions in Top- $k(p)$ with larger positions can be easily answered by the answer of Top- $k$ on the former traversed nodes. Now let $(1, x)$ and $(2, y)$ be the first or second candidates in such unvisited or half-visited node $p$, which can be found by Top- $k\left(a, b, A_{1}\right)$ and Top- $k\left(a, b, A_{2}\right)$.

If one of the candidates is already picked before at some node $p^{\prime}$ (without loss of generality, assume that $(1, x)$ is picked by $\left.p^{\prime}\right)$ and $y \in p^{\prime}$, we can know $A[1][x]>A[2][y]$ with no extra information. If there is no such node we read next 1 bit to decode. Since $X$ is encoded in the modified level order, one can easily show that such bit is encoded for pick $(i, j)$ at $p$. Therefore, we can encode to answer Top- $k(1,2, i, j, A)$ queries at most $4 n$ extra bits, if we can answer sorted 2 -sided Top- $k$ queries on $A_{1}$ and $A_{2}$.

For the special case when $k=2$, the following theorem shows that the space bound of the encoding of Theorem 3 can be improved.

Theorem 4 Given a $2 \times n$ array $A$, if there exists an $S(n)$-bit encoding to answer sorted 2-sided Top-2 queries on a $1 D$ array of size $n$ in $T(n)$ time and such encoding 
can be constructed in $C(n)$ time, then we can encode $A$ in $2 S(n)+3 n$ bits using $O\left(C(n)+n^{2}+n T(n)\right)$ time, for answering Top-2 queries on $A$.

Proof It is enough to show that the bit string $X$, defined in the proof of Theorem 3 , has length at most $3 n$ when it is constructed under $D_{A}^{2}$. We claim that after all nodes in $D_{A}^{2}$ are traversed in modified level-order, $i$-th column is picked (i.e., any position in the $i$-th column is picked) at most three times for all $1 \leq i \leq n$, which proves the theorem.

To prove the claim, let $f(i)$ (respectively, $s(i)$ ) be the position of the larger (respectively, smaller) element between $(1, i)$ and $(2, i)$, and suppose $p$ with label $I_{p}=[a, b]$ be the first node in the modified level order at which the position $s(i)$ is picked for the first time. Then by the traversing algorithm and definition of $D_{A}^{2}, f(i)$ is already picked before $s(i)$ is picked. This implies that Top-2(p) $=\{f(i), s(i)\}$ and the $i$-th column is not contained in all descendants of $p$ (note that the labels of $p$ 's children are $[a, i-1]$ and $[i+1, b]$ by the definition of $\left.D_{A}^{2}\right)$. Also, we claim that $s(i)$ is not picked at any other node $p^{\prime}$ with label $I_{p^{\prime}}=\left[a^{\prime}, b^{\prime}\right]$ where $I_{p} \not \subset I_{p^{\prime}}$ and $I_{p^{\prime}} \not \subset I_{p}$. To prove this, suppose that $s(i)$ is picked at the node $p^{\prime}$, and without loss of generality, $p^{\prime}$ precedes the node $p$. Then by the definition of $D_{A}^{2}$, the element in $f(a-1)$ is larger than $f(i) \in$ Top-2 $\left(p^{\prime}\right) \cap$ Top-2 $(p)$ (note that the ancestor of $p$ picks $f(a-1)$, to have $p$ as descendant). This implies $s(i) \notin$ Top-2( $\left.p^{\prime}\right)$ and hence $s(i)$ cannot picked at the node $p^{\prime}$. Thus, $s(i)$ is only picked once at the node $p$, and $f(i)$ can be picked at most twice by Lemma 3, which implies any column is picked at most three times.

From the encoding of Theorem 3, the following theorem shows that we can obtain an encoding for answering sorted 4-sided Top- $k$ queries on an $m \times n$ array by extending the encoding of a $2 \times n$ array.

Theorem 5 Given an $m \times n$ array $A$, if there exists an $S(n, k)$-bit encoding to answer sorted 2-sided Top- $k$ queries on a $1 D$ array of size $n$, then we can encode $A$ in $m S(n, k)+2 n m(m-1)$ bits, to support sorted 4-sided Top-k queries on A.

Proof For $1 \leq i \leq j \leq n$ and $1 \leq a \leq b \leq m$, we first use $m S(n, k)$ bits to support sorted 2-sided Top-k(a,a,i,j,A) queries. Also we encode the answer Top- $k\left(1,2, i, j, A_{a b}\right)$ queries on $2 \times n$ array $A_{a b}$, whose first and second row are $a$-th and $b$-th row in $A$ respectively. By Theorem 3, we can encode such queries on all possible $A_{a b}$ arrays using $2 n m(m-1)$ extra bits. For $1 \leq a \leq m$ and $1 \leq \ell \leq k$, let $\alpha_{i j}(a, \ell)$ be the position of $\ell$-th largest element in $A[a][i \ldots j]$ Note that we can find such $\alpha_{i j}(a, \ell)$ using Top- $k(a, a, i, j, A)$ query.

Now we describe how to answer Top- $k(a, b, i, j, A)$ query. We first define $(b-a+1)$ values $c_{a} \ldots c_{b}$ and set $c_{a}=c_{a+1} \cdots=c_{b}=1$. After that, we find a position of largest value in $A[a \ldots b][i \ldots j]$ by comparing $\alpha_{i j}\left(a, c_{a}\right), \alpha_{i j}\left(a+1, c_{a+1}\right) \ldots \alpha_{i j}\left(b, c_{b}\right)$ and find a position with largest element among them. It is clear that for $a \leq a^{\prime} \leq b^{\prime} \leq b$, we can compare values at the position $\alpha_{i j}\left(a^{\prime}, c_{a^{\prime}}\right)$ and $\alpha_{i j}\left(b^{\prime}, c_{b^{\prime}}\right)$ using Top- $k\left(1,2, i, j, A_{a^{\prime} b^{\prime}}\right)$ query since at least one of the their corresponding positions in $A_{a^{\prime} b^{\prime}}$ is an answer of Top- $k\left(1,2, i, j, A_{a^{\prime} b^{\prime}}\right)$ query. Suppose for $a \leq a^{\prime} \leq b$, 
$\alpha_{i j}\left(a^{\prime}, c_{a^{\prime}}\right)$ a position with the largest value in $A[a \ldots b][i \ldots j]$. Then we increase $c_{a^{\prime}}$ by 1 , and compare $\alpha_{i j}\left(a^{\prime}, c_{a^{\prime}}\right)$ and $\alpha_{i j}\left(b^{\prime}, c_{b^{\prime}}\right)$ again to find a position of the second largest value in $A[a \ldots b][i \ldots j]$. We do this procedure iteratively until we find a position of $k$-th largest value in $A[a \ldots b][i \ldots j]$.

Corollary 1 Given an $m \times n$ array $A$, if there exists an $S(n)$-bit encoding to answer sorted 2-sided Top-2 queries on a $1 D$ array of size $n$, then we can encode $A$ in $m S(n)+1.5 n m(m-1)$ bits, to support sorted 4-sided Top-2 queries on A.

Finally, if we combine the encoding of Theorem 5 and Gawrychowski and Nicholson's $\left(\lg \left(\begin{array}{c}(k+1) n \\ n\end{array}\right)+o(n)\right)$-bit optimal encoding for sorted 2-sided Top- $k$ queries on a $1 \mathrm{D}$ array [9], we obtain an encoding as follows.

Corollary 2 Given an $m \times n$ array $A$, there exists an $\left(m \lg \left(\begin{array}{c}(k+1) n \\ n\end{array}\right)+2 n m(m-1)+o(n)\right)$-bit encoding, to support sorted 4-sided Top- $k$ queries on A. Also when $k=2$, there exists an $\left(m \lg \left(\begin{array}{c}3 n \\ n\end{array}\right)+1.5 n m(m-1)+o(n)\right)$-bit encoding, to support sorted 4-sided Top-2 queries on A.

\section{Data Structure for 4-Sided Top-k Queries on $2 \times n$ Array}

The encoding of Theorem 3 shows that $4 n$ bits are sufficient for answering Top- $k$ queries whose range spans both rows, when encodings for answering sorted 2-sided Top- $k$ queries for each row are given. However, this encoding does not support queries efficiently (takes $O\left(k^{2} n^{2}+k n T(n, k)\right)$ time) since we need to reconstruct all the nodes in $D_{A}$ to answer a query (in the worst case). We now show that the query time can be improved to $O\left(k^{2}+k T(n, k)\right)$ time if we use $(4 k+7) n+k o(n)$ additional bits. Note that if we simply use the data structure of Grossi et al. [11] (which takes $44 n \lg k+O(n \lg \lg k)$ bits to encode a $1 \mathrm{D}$ array of length $n$ to support Top- $k$ queries in $O(k)$ time) on the 1D array of size $2 n$ obtained by writing the values of $A$ in column-major order, we can answer Top- $k$ queries on $A$ in $O(k)$ time using $88 n \lg k+O(n \lg \lg k)$ additional bits. Although our data structure takes more query time and takes asymptotically more space, it uses less space for small values of $k$ (note that $4 k+7<88 \lg k$ for all integers $2 \leq k<160$ ) when $n$ is sufficiently large. We now describe our data structure.

We first define a graph $G_{12}=\left(V\left(G_{12}\right), E\left(G_{12}\right)\right)$ on $A$ as follows. The set of vertices $V\left(G_{12}\right)=\{1,2, \ldots n\}$, and there exists an edge $(i, j) \in E\left(G_{12}\right)$ if and only if (i) $i<j$ and $A[1][i]<A[2][j]$, (ii) there are at most $k-1$ positions in $A[1,2][i \ldots j]$ whose corresponding values are larger than both $A[1][i]$ and $A[2][j]$, and (iii) there is no vertex $j^{\prime} \in\{i+1, i+2, \ldots, n\}$ where $A[1][i]<A[2]\left[j^{\prime}\right]<A[2][j]$ and satisfies the condition (ii). We also define a graph $G_{21}$ on $A$ which is analogous to $G_{12}$, by replacing $A[1][i], A[2][j]$ and $A[2]\left[j^{\prime}\right]$ with $A[2][i] A[1][j]$, and $A[1]\left[j^{\prime}\right]$, respectively in all three conditions. Each of the graphs $G_{12}$ and $G_{21}$ have $n$ vertices and at most $n$ edges. Also for any vertex $v \in V\left(G_{12}\right)$ (resp., $V\left(G_{21}\right)$ ), there exists at most one vertex $v^{\prime}$ in $G_{12}$ (resp., $G_{21}$ ) such that $v$ is incident to $v^{\prime}$ and 


\begin{tabular}{|c|c|c|c|c|c|c|c|c|c|}
\hline$A_{1}$ & 1 & 21 & 17 & 12 & 20 & 3 & 15 & 11 & 10 \\
\hline$A_{2}$ & 6 & 5 & 16 & 14 & 19 & 2 & 18 & 4 & 7 \\
\hline
\end{tabular}
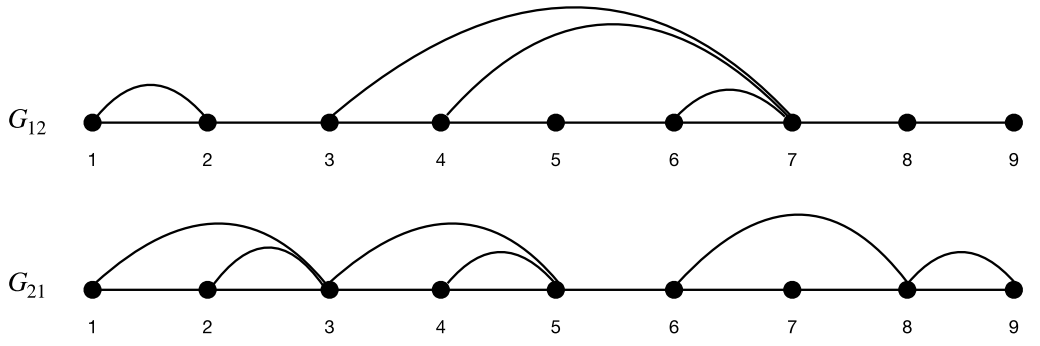

$$
\begin{aligned}
& P_{A}=1 \quad 00011000100 \\
& Q_{12}=\begin{array}{llllllll}
0 & 1 & 0 & 0 & 1 & 0 & 1 & 1
\end{array} \\
& Q_{21}=\begin{array}{lllllllll}
0 & 0 & 0 & 0 & 1 & 0 & 1 & 0
\end{array}
\end{aligned}
$$

Fig. $3 G_{12}, G_{21}, P_{A}, Q_{12}$, and $Q_{21}$ to support Top-3 queries on $2 \times n$ array $A$

$v<v^{\prime}$. See Fig. 3 for an example. We now show that $G_{12}$ (thus, also $G_{21}$ ) is a $k$-page graph, i.e. there exist no $k+1$ edges $\left(i_{1}, j_{1}\right) \ldots\left(i_{k+1}, j_{k+1}\right) \in E\left(G_{12}\right)$ such that $i_{1}<i_{2} \cdots<i_{k+1}<j_{1}<j_{2} \cdots<j_{k+1}$.

Lemma 4 Given $2 \times n$ array $A$, a graph $G_{12}$ on $A$ is k-page graph.

Proof Suppose that there are $k+1$ edges $\left(i_{1}, j_{1}\right) \ldots\left(i_{k+1}, j_{k+1}\right) \in E\left(G_{12}\right)$ such that $i_{1}<i_{2} \cdots<i_{k+1}<j_{1}<j_{2} \cdots<j_{k+1}$, and for $1 \leq t \leq k+1$, let $i_{t}$ be a position of the minimum element in $A_{1}\left[i_{1} \ldots i_{k+1}\right]$. Then by the definition fo $G_{12}$, there are at least $k$ positions $\left(1, i_{t+1}\right), \ldots,\left(1, i_{k+1}\right),\left(2, j_{1}\right), \ldots,\left(2, j_{t-1}\right)$ in $A[1,2]\left[i_{t} \ldots j_{t}\right]$ whose corresponding values in $A$ are larger than both $A[1]\left[i_{t}\right]$ and $A[2]\left[j_{t}\right]$, which contradicts the definition of $G_{12}$.

From the above lemma and the succinct representation of $k$-page graphs of Munro and Raman [18] (with minor modification as described in [8]), we can encode $G_{12}$ and $G_{21}$ using $(4 k+4) n+k \cdot o(n)$ bits in total, and for any vertex $v$ in $V\left(G_{12}\right) \cup V\left(G_{21}\right)$, we can find a vertex with the largest index which incident to $v$ in $O(k)$ time. Also to compare the elements in the same column, we maintain a bit string $P_{A}[1 \ldots n]$ of size $n$ such that for $1 \leq i \leq n, P_{A}[i]=0$ if and only if $A[1][i]>A[2][i]$. Finally, for $G_{12}$ (resp., $G_{21}$ ), we maintain another bit string $Q_{12}[1 \ldots n-1]$ (resp., $Q_{21}[1 \ldots n-1]$ ) such that for $1 \leq i \leq n-1, Q_{12}[i]=1$ (resp., $\left.Q_{21}[i]=1\right)$ if and only if all elements in $A_{2}[i+1 \ldots n]$ (resp., $\left.A_{1}[i+1 \ldots n]\right)$ are smaller than $A[1][i]$ (resp., $A[2][i]$ ) (see Fig. 3 for an example). We now show that if there is an encoding which can answer the sorted Top- $k$ queries on each row, then the encoding of $G_{12}, G_{21}$, and the additional arrays defined above are enough to answer 4-sided Top- $k$ queries on $A$. 
Theorem 6 Given a $2 \times n$ array $A$, if there exists an $S(n, k)$-bit encoding to answer sorted 2-sided Top- $k$ queries on a $1 D$ array of size $n$ in $T(n, k)$ time, then there is a $2 S(n, k)+(4 k+7) n+k \cdot o(n)$-bit data structure which can answer Top- $k$ queries on $A$ in $O\left(k^{2}+k T(n, k)\right)$ time.

Proof For $1 \leq i \leq j \leq n$, we first use $2 S(n, k)$ bits to support sorted 2-sided Top- $k(1,1, i, j, A)$ and Top- $k(2,2, i, j, A)$ queries in $T(n, k)$ time.

To answer Top- $k(1,2, i, j, A)$ query, we maintain succinct representations of $G_{12}$ and $G_{21}[8,18]$ using $(4 k+4) n+k o(n)$ bits, and $P_{A}, Q_{12}$, and $Q_{21}$ using $3 n$ bits. Now for $1 \leq p \leq k$, let $a_{p}$ (resp., $b_{p}$ ) be the position of the $p$-th largest value in $A_{1}[i \ldots j]$ (resp., $\left.A_{2}[i \ldots j]\right)$, which can be answered in $O(T(n, k))$ time using the encoding of Top- $k$ queries on each row.

We first find the position of the largest value in $A[1,2][i \ldots j]$ by comparing $A[1]\left[a_{1}\right]$ and $A[2]\left[b_{1}\right]$. If $A[1]\left[a_{1}\right]<A[2]\left[b_{1}\right]\left(\right.$ resp., $\left.A[1]\left[a_{1}\right]>A[2]\left[b_{1}\right]\right)$, we compare $A[1]\left[a_{1}\right]$ with $A[2]\left[b_{2}\right]$ (resp., $A[1]\left[a_{2}\right]$ with $A[2]\left[b_{1}\right]$ ) to find the position of the second-largest value in $A[1,2][i \ldots j]$. By repeating this procedure iteratively $k$ times, we can answer the Top- $k(1,2, i, j, A)$ query.

Now we describe how to compare $A[1]\left[a_{p}\right]$ with $A[2]\left[b_{q}\right]$, for all $p+q \leq k+1$ (in the above procedure, we do not need to compare $A[1]\left[a_{p}\right]$ with $A[2]\left[b_{q}\right]$ if $p+q>k+1)$. If $a_{p}=b_{q}$, the result of the comparison is already stored in the bit $P_{A}\left[a_{p}\right]$. Now suppose that $a_{p}<b_{q}$ (if $a_{p}>b_{q}$, we use $G_{21}$ and $Q_{21}$ instead of $G_{12}$ and $Q_{12}$ respectively, in the following procedure), and let $a_{p}^{\prime}$ be a vertex with the largest index in $G_{12}$ which is incident to $a_{p}$, if it exists. Note that we can find such $a_{p}^{\prime}$ in $O(k)$ time $[8,18]$. If there is no vertex incident to $a_{p}$ or $a_{p}^{\prime}<a_{p}$, we show that $A[1]\left[a_{p}\right]>A[2]\left[b_{q}\right]$ by considering the following two cases.

- (i) $Q_{12}\left[a_{p}\right]=1$ : From the definition of $Q_{12}$, it follows that $A[1]\left[a_{p}\right]>A[2]\left[b_{q}\right]$.

- (ii) $Q_{12}\left[a_{p}\right]=0$ : In this case, (a) $A[1]\left[a_{p}\right]<A[2]\left[b_{q}\right]$, but there are at least $k$ positions in $A[1,2]\left[a_{p} \ldots b_{q}\right]$ whose corresponding values are larger than both $A[1]\left[a_{p}\right]$ and $A[2]\left[b_{q}\right]$ or (b) $A[1]\left[a_{p}\right]>A[2]\left[b_{q}\right]$. However (a) cannot hold since there are at most $(p-1)+(q-1) \leq k-1$ positions in $A[1,2]\left[a_{p} \ldots b_{q}\right]$ whose corresponding values are larger than both $A[1]\left[a_{p}\right]$ and $A[2]\left[b_{q}\right]$. Therefore $A[1]\left[a_{p}\right]>A[2]\left[b_{q}\right]$.

Now consider the case $a_{p}<a_{p}^{\prime}$. If $a_{p}^{\prime} \leq b_{q}$, then $A[1]\left[a_{p}\right]<A[2]\left[b_{q}\right]$ if and only if $A[2]\left[a_{p}^{\prime}\right]<A[2]\left[b_{q}\right]$ by the definition of $G_{12}$. If $a_{p}^{\prime}>b_{q}$, we first compare $A[2]\left[a_{p}^{\prime}\right]$ with $A[2]\left[b_{q}\right]$. If $A[2]\left[a_{p}^{\prime}\right]<A[2]\left[b_{q}\right]$, then $A[1]\left[a_{p}\right]<A[2]\left[b_{q}\right]$ by the definition of $G_{12}$. If not, (a) $A[1]\left[a_{p}\right]<A[2]\left[b_{q}\right]$, but there are at least $k$ positions in $A[1,2]\left[a_{p} \ldots b_{q}\right]$ whose corresponding value is larger than both $A[1]\left[a_{p}\right]$ and $A[2]\left[b_{q}\right]$, or (b) $A[1]\left[a_{p}\right]>A[2]\left[b_{q}\right]$. However, (a) cannot hold by the same reason as the case when there is no vertex incident to $a_{p}$ or $a_{p}^{\prime}<a_{p}$, and $Q_{12}\left[a_{p}\right]=0$. Therefore $A[1]\left[a_{p}\right]>A[2]\left[b_{q}\right]$ if $A[2]\left[a_{p}^{\prime}\right]>A[2]\left[b_{q}\right]$. Also since $\left(2, b_{q}\right)$ is one of the answers of Top- $k\left(i, j, A_{2}\right)$ query, we can compare $A[2]\left[a_{p}^{\prime}\right]$ with $A[2]\left[b_{q}\right]$ in $T(n, k)$ time using the Top- $k$ encoding on the second row. By the procedure describe above, each iteration step takes at most $O(k+T(n, k))$ time, thus we can answer Top- $k(1,2, i, j . A)$ query in $O\left(k^{2}+k T(n, k)\right)$ time. 


\section{Lower Bounds for Encoding Range Top-k Queries on $2 \times n$ Array}

In this section, we consider the lower bound on space for encoding a $2 \times n$ array $A$ to support unsorted 1-sided and sorted 4-sided Top- $k$ queries, when $k>1$. Specifically for $1 \leq i \leq j \leq n$, we consider to lower bound on extra space for answering (i) unsorted Top- $k(1,2,1, i)$ queries, assuming that we have access to the encodings of the individual rows of $A$ that can answer unsorted 1-sided (or 2-sided) Top- $k$ queries, and (ii) sorted Top- $k(1,2, i, j)$ queries, assuming that we have access to the encodings of the individual rows of $A$ that can answer sorted 2-sided Top- $k$ queries. We show that for answering unsorted 1-sided queries on $A$, at least $1.27 n-o(n)($ or $2 n-O(\lg n))$ extra bits are necessary, and for answering unsorted or sorted 4-sided queries on $A$, at least $2 n-O(\lg n)$ extra bits are necessary.

For simplicity (to avoid writing floors and ceilings, and to avoid considering some boundary cases), we assume that $k$ is even. (Also, if $k$ is odd we can consider the lower bound on extra space for answering 4-sided Top- $k$ queries as the lower bound of extra space for answering 4-sided Top- $(k-1)$ queriesit is clear that former one requires more space.) For both unsorted and sorted query cases, we assume that all elements in $A$ are distinct, and come from the set $\{1,2, \ldots 2 n\}$; and also that each row in $A$ is sorted in the ascending order. Finally, for $1 \leq \ell \leq 2 n$, we define the mapping $A^{-1}(\ell)=(i, j)$ if and only if $A[i][j]=\ell$.

Unsorted 1-sided Top- $k$ query The following theorem gives the lower bound for answering unsorted 1-sided Top- $k$ queries on $2 \times n$ array $A$, when the encodings for answering unsorted 1-sided (or 2-sided) Top- $k$ queries on both rows are already given. Note that this lower bound also gives the lower bound for answering unsorted 4-sided Top- $k$ queries on $2 \times n$ array under the same condition.

Theorem 7 Given a $2 \times n$ array $A$ and encodings for answering unsorted 1-sided (or 2-sided) Top- $k$ queries on both rows in A, at least $\lceil(n-k / 2) \lg (1+\sqrt{2})\rceil-o(n)=1.27(n-k / 2)-o(n)$ additional bits are necessary for answering unsorted 1 -sided Top- $k$ queries on $A$.

Proof If $n \leq k / 2$ we do not need any extra space since all positions are answers of unsorted Top- $k(1,2,1, i, A)$ queries for $i \leq n$. Now suppose that $n>k / 2$. In this case, let $U_{i}$ be a set of all possible arrays of size $2 \times n$ which satisfies the following properties:

- For any $B \in U_{i}$, all of $\{1,2 \ldots 2 i\}$ are in $B[1,2][1 \ldots i]$ and each row in $B$ is sorted in the ascending order (thus, all the arrays in $U_{i}$ have same encodings for answering unsorted 1 and 2-sided Top- $k$ queries on their individual rows), and

- for any two distinct arrays $B, C \in U_{i}$, there exists $1 \leq j \leq i$ such that $\left\{B^{-1}(2 j-1), B^{-1}(2 j)\right\} \neq\left\{C^{-1}(2 j-1), C^{-1}(2 j)\right\}$.

By the definition of $U_{i}$, for any $1 \leq i \leq n-k / 2$ and two distinct arrays $B, C \in U_{i}$, there exists a position $1 \leq j \leq i$ where $B$ and $C$ have distinct answers of unsorted 
Top- $k(1,2,1, k / 2+j)$ queries, which implies $\log \left|U_{n}\right|$ gives the lower bound of additional space for answering the 1 -sided Top- $k$ queries on $2 \times n$ array. We compute the size of $U_{i}$ as follows. $\left|U_{1}\right|=1$ since there exists only one case as $\left\{B^{-1}(1), B^{-1}(2)\right\}=\{(1,1),(2,1)\}$. For $i=2$, we can consider three cases as $(1,2,3,4),(1,3,2,4)$, or $(1,4,2,3)$ if we write a elements of $B[1,2][1,2]$ for $B \in U_{2}$ in row-major order (note that each row is sorted in ascending order).

Next, consider the case when $2<i \leq n-k / 2$. In this case for any $B \in U_{i-1}$, $\left\{B^{-1}(2 i-3), B^{-1}(2 i-2)\right\} \subset\{\{(1, i),(2, i)\},\{(1, i-1),(1, i)\},\{(2, i-1),(2, i)\}\}$. To construct arrays in $U_{i}$, we construct a set $E_{B} \subset U_{i}$ from the array in $B \in U_{i-1}$ such that for any $B_{1}, B_{2} \in E_{B}$ and $1 \leq j<i+k / 2$, $\operatorname{Top}-k\left(1,2,1, j, B_{1}\right)=\operatorname{Top}-k\left(1,2,1, j, B_{2}\right)=\operatorname{Top}-k(1,2,1, j, B) \quad$ and Top- $k\left(1,2,1, i+k / 2, B_{1}\right) \neq \operatorname{Top}-k\left(1,2,1, i+k / 2, B_{2}\right)$. It is clear that $\left|U_{i}\right|=\sum_{B \in U_{i-1}}\left|E_{B}\right|$. Now we consider two cases as follows.

- Case 1. $B^{-1}(2 i-3)$ and $B^{-1}(2 i-2)$ are in different rows: In this case, for any $C \in E_{B}$ the position of the first and the second largest value in $C[1,2][1 \ldots 2(i-1)]$ are $(1, i-1)$ and $(2, i-1)$ respectively and for any $B_{1}, B_{2} \in E_{B},\left\{B_{1}^{-1}(2 i-1), B_{2}^{-1}(2 i)\right\}$ are distinct. In this case, $\left|E_{B}\right|=3$ since only $\{(1, i),(2, i)\},\{(1, i-1),(1, i)\}$, or $\{(2, i-1),(2, i)\}$ can be $\left\{B^{-1}(2 i-1), B^{-1}(2 i)\right\}$ for any array $C \in E_{B}$ respectively, which satisfy the above condition and maintaining both rows in $C$ as sorted in ascending order. Furthermore, since both $B^{-1}(2 i-3)$ and $B^{-1}(2 i-2)$ are in $(i-1)$-th column, the number of $B \in U_{i-1}$ in this case is $\left|U_{i-2}\right|$.

- Case 2. $B^{-1}(2 i-3)$ and $B^{-1}(2 i-2)$ are in the same row: Without loss of generality, assume that both $B^{-1}(2 i-3)$ and $B^{-1}(2 i-2)$ are in the first row. Then for any $C \in E_{B}$ the position of the first and the second largest value in $C[1,2][1 \ldots 2(i-1)]$ are $(1, i-2)$ and $(1, i-1)$ respectively, and for any $B_{1}, B_{2} \in E_{B},\left\{B_{1}^{-1}(2 i-1), B_{2}^{-1}(2 i)\right\}$ are distinct. Therefore $\left|E_{B}\right|=2$ in this case since only $\{(1, i),(2, i)\}$ or $\{(1, i-1),(1, i)\}$ can be $\{C(2 i-1), C(2 i)\}$ for any array $C \in E_{B}$ respectively which satisfy the above condition and maintaining both rows in $C$ as sorted in ascending order. Also since all the $B$ is in the Case 1 or 2, the number of $B \in U_{i-1}$ in this case is $\left|U_{i-1}-U_{i-2}\right|$.

By the statement described above, we obtain a recursive relation $\left|U_{i}\right|=3\left|U_{i-2}\right|+2\left(\left|U_{i-1}\right|-\left|U_{i-2}\right|\right)$. By solving the recursive relation using characteristic equation, we obtain $\left|U_{i}\right| \leq(1+\sqrt{2})^{i}-o(n)$, which proves the theorem.

Sorted and 4-sided Top- $k$ query In this case we divide a $2 \times n$ array $A$ into $2 n / k$ blocks $A_{1} \ldots A_{2 n / k}$ of size $2 \times k / 2$ as for $1 \leq \ell \leq k / 2, A_{\ell}[i][j]=A[i][2(\ell-1)+j]$ and all values of $A_{\ell}$ are in $\{k(\ell-1)+1 \ldots k \ell\}$. Then for any $2 \times n$ array $A$ and $A^{\prime}$, sorted Top- $k(1,2, k(i-1) / 2+1, k i / 2, A) \neq \operatorname{Top}-k\left(1,2, k(i-1) / 2+1, k i / 2, A^{\prime}\right)$, and $\operatorname{Top}-k(1,2,1, k i / 2, A) \neq \operatorname{Top}-k\left(1,2,1, k i / 2, A^{\prime}\right)$, if there exists a position $1 \leq i \leq 2 n / k$ where $A_{i} \neq A_{i}^{\prime}$. Let $S_{i}$ be the set of all possible arrays of size $2 \times i$ such that for any $B \in S_{i}$, all values of $B$ are in $\{1,2 i\}$ and both rows of $B$ are sorted in ascending order, which implies all the arrays in $S_{i}$ have same encodings for answering unsorted and sorted 2 -sided Top- $k$ queries on their individual rows. Since the 
size of $S_{i}$ is same as central binomial number, $\left(\begin{array}{c}2 i \\ i\end{array}\right)$, which is well-known as at least $4^{i} / \sqrt{4 i}$ [16]. Therefore, at least $\left\lceil 2 n \lg \left|S_{k / 2}\right| / k\right\rceil \geq 2 n-O(\lg n)$ additional bits are necessary for answering sorted Top- $k$ queries that span both the rows, when encodings for answering sorted (or unsorted) on both rows are given.

Theorem 8 Given a $2 \times n$ array $A$, at least $2 n-O(\lg n)$ additional bits are necessary for answering sorted 4-sided Top- $k$ queries on $A$ if encodings for answering sorted (or unsorted) 2-sided Top- $k$ queries on both rows in A are given.

\section{Conclusions and Open Problems}

In this paper, we proposed encodings for answering Top- $k$ queries on 2D arrays. For $2 \times n$ arrays, we proposed upper and lower bounds on space for answering sorted and unsorted 4-sided Top- $k$ queries. Finally, we obtained an $\left(m \lg \left(\begin{array}{c}(k+1) n \\ n\end{array}\right)+2 n m(m-1)+o(n)\right)$-bit encoding for answering 4-sided sorted Top- $k$ queries on $m \times n$ arrays. We end with the following open problems:

- Can we support 4-sided sorted Top- $k$ queries with efficient query time on $m \times n$ arrays using less than $O(n m \lg n)$ bits when $m=o(\lg n)$ ?

- Can we obtain an improved lower or upper bound for answering 4-sided sorted Top- $k$ queries on $2 \times n$ arrays?

Funding Open access funding provided by NTNU Norwegian University of Science and Technology (incl St. Olavs Hospital - Trondheim University Hospital).

Open Access This article is licensed under a Creative Commons Attribution 4.0 International License, which permits use, sharing, adaptation, distribution and reproduction in any medium or format, as long as you give appropriate credit to the original author(s) and the source, provide a link to the Creative Commons licence, and indicate if changes were made. The images or other third party material in this article are included in the article's Creative Commons licence, unless indicated otherwise in a credit line to the material. If material is not included in the article's Creative Commons licence and your intended use is not permitted by statutory regulation or exceeds the permitted use, you will need to obtain permission directly from the copyright holder. To view a copy of this licence, visit http://creativecommons.org/ licenses/by/4.0/.

\section{References}

1. Berkman, O., Vishkin, U.: Recursive star-tree parallel data structure. SIAM J. Comput. 22(2), 221242 (1993)

2. Brodal, G.S., Brodnik, A., Davoodi, P.: The encoding complexity of two dimensional range minimum data structures. In: Algorithms-ESA 2013-21st Annual European Symposium, Sophia Antipolis, France, September 2-4, 2013. Proceedings, pp. 229-240 (2013)

3. Brodal, G.S., Davoodi, P., Rao, S.S.: On space efficient two dimensional range minimum data structures. Algorithmica 63(4), 815-830 (2012) 
4. Brodal, G.S., Fagerberg, R., Greve, M., López-Ortiz, A.: Online sorted range reporting. In: ISAAC 2009, Proceedings, pp. 173-182 (2009)

5. Davoodi, P., Navarro, G., Raman, R., Rao, S.S.: Encoding range minima and range top-2 queries. Philos Trans R Soc Lond Math Phys Eng Sci (2014). https://doi.org/10.1098/rsta.2013.0131

6. Fischer, J., Heun, V.: Finding range minima in the middle: approximations and applications. Math. Comput. Sci. 3(1), 17-30 (2010)

7. Frederickson, G.N.: An optimal algorithm for selection in a min-heap. Inf. Comput. 104(2), 197214 (1993)

8. Gawrychowski, P., Nicholson, P.K.: Encodings of range maximum-sum segment queries and applications. In: Combinatorial Pattern Matching-26th Annual Symposium, CPM 2015, Ischia Island, Italy, June 29-July 1, 2015, Proceedings, pp. 196-206 (2015)

9. Gawrychowski, P., Nicholson, P.K.: Optimal encodings for range top-k k, selection, and min-max. In: Automata, Languages, and Programming - 42nd International Colloquium, ICALP 2015, Kyoto, Japan, July 6-10, 2015, Proceedings, Part I, pp. 593-604 (2015)

10. Golin, M.J., Iacono, J., Krizanc, D., Raman, R., Satti, S.R., Shende, S.M.: Encoding 2d range maximum queries. Theor. Comput. Sci. 609, 316-327 (2016)

11. Grossi, R., Iacono, J., Navarro, G., Raman, R., Rao, S.S.: Asymptotically optimal encodings of range data structures for selection and top-k queries. ACM Trans. Algorithms 13(2), 28:1-28:31 (2017)

12. Grossi, R., Iacono, J., Navarro, G., Raman, R., Satti, S.R.: Encodings for range selection and top-k queries. ESA 2013, 553-564 (2013)

13. He, M.: Succinct and implicit data structures for computational geometry. In: Space-Efficient Data Structures, Streams, and Algorithms-Papers in Honor of J. Ian Munro on the Occasion of His 66th Birthday, pp. 216-235 (2013)

14. Jo, S., Lingala, R., Satti, S.R.: Encoding two-dimensional range top-k queries. In: 27th Annual Symposium on Combinatorial Pattern Matching, CPM 2016, June 27-29, 2016, Tel Aviv, Israel, pp. 3:1-3:11 (2016)

15. Jo, S., Satti, S.R.: Encoding two-dimensional range top-k queries revisited. In: 29th International Symposium on Algorithms and Computation, ISAAC 2018, December 16-19, 2018, Jiaoxi, Yilan, Taiwan, pp. 69:1-69:13 (2018)

16. Kazarinoff, N.: Geometric Inequalities. Random House, New York (1961)

17. Miltersen, P.B.: Cell probe complexity-a survey. FSTTCS (1999)

18. Munro, J.I., Raman, V.: Succinct representation of balanced parentheses and static trees. SIAM J. Comput. 31(3), 762-776 (2001)

19. Rahul, S., Tao, Y.: On top-k range reporting in $2 d$ space. In: Proceedings of the 34th ACM Symposium on Principles of Database Systems, PODS 2015, Melbourne, Victoria, Australia, May 31-June 4, 2015, pp. 265-275 (2015)

20. Rahul, S., Tao, Y.: Efficient top-k indexing via general reductions. In: Proceedings of the 35th ACM SIGMOD-SIGACT-SIGAI Symposium on Principles of Database Systems, PODS 2016, San Francisco, CA, USA, June 26-July 01, 2016, pp. 277-288 (2016)

Publisher's Note Springer Nature remains neutral with regard to jurisdictional claims in published maps and institutional affiliations. 